\title{
Atmospheric infrasound generation by ocean waves in finite depth: unified theory and application to radiation patterns
}

\author{
M. De Carlo ${ }^{\oplus}, 1,2$ F. Ardhuin ${ }^{2,3}$ and A. Le Pichon ${ }^{1}$ \\ ${ }^{1}$ CEA, DAM, DIF F-91297 Arpajon, France \\ ${ }^{2}$ Univ. Brest, CNRS, IRD, Ifremer, Laboratoire d'Océanographie Physique et Spatiale (LOPS), IUEM, F-29238 Brest, France. \\ E-mail: Marine.DECARLO@cea.fr \\ ${ }^{3}$ Marine Physical Laboratory, Scripps Institution of Oceanography, La Jolla, CA 92037, USA
}

Accepted 2020 January 8. Received 2019 October 28; in original form 2019 June 7

\begin{abstract}
SUMMAR Y
Between 0.1 and $0.5 \mathrm{~Hz}$, infrasound signals recorded in the atmosphere are dominated by ocean-generated noise called microbaroms. Microbaroms propagate through the atmosphere over thousands of kilometres due to low absorption and efficient ducting between the ground and the stratopause. Different theoretical models have been developed to characterize the source of microbaroms, all based on the second-order nonlinear interaction of ocean waves. While early theories considered an infinite ocean depth and a source radiation depending on the acoustic wave elevation angle, other works have approximated the radiation pattern as a monopole, and found a considerable effect of the water depth. This paper reviews these models and extends the previous theories to the combined effects of both finite depth ocean and source directivity in both elevation and azimuth angles. It is found that the water depth has a negligible effect for the near-horizontally propagating acoustic waves that should dominate the measured microbarom records. Another important result is that the microbarom azimuthal variation can be highly directive locally, but it generally becomes isotropic when integrated over a realistic source region.
\end{abstract}

Key words: Interface waves; Wave propagation; Infrasound.

\section{INTRODUCTION}

Continuous oscillations of the ground displacement and atmospheric pressure, named respectively secondary microseisms and microbaroms, are measured worldwide by seismological and infrasound networks with a dominant frequency around $0.2 \mathrm{~Hz}$ (Benioff \& Gutemberg 1939). They are generated by second-order nonlinear interaction of ocean gravity waves of similar frequency propagating in almost opposite directions (Longuet-Higgins 1950; Hasselmann 1963).

Microbarom propagates through the atmosphere over large distances due to low absorption rates and efficient atmospheric ducting between the ground and the stratopause (Drob 2019; Waxler \& Assink 2019). Studying microbaroms recorded for 4 yr at Palisades, New York, Donn \& Rind (1971) have revealed the importance of winds in the higher atmosphere for their propagation, pointing to the capability of ground-based measurements to probe the higher atmosphere, for which very few other observations are available.

Recent developments of infrasound networks at global and continental scales facilitate the analysis of acoustic waves for probing unresolved atmospheric structures in the middle atmosphere (Blanc et al. 2018; Marty 2019). This has motivated mathematical developments of geophysical inverse problems using infrasound from well-identified sources (Drob et al. 2010; Assink et al. 2014). Ducting of infrasound depends on the 3-D wind and temperature fields and is most efficient if the propagation direction coincides with the polar vortex at midlatitude regions. In particular, the main characteristics of Sudden Stratospheric Warming events have been successfully derived from directional microbarom amplitude variations resulting from changes in stratospheric and thermospheric propagation conditions (e.g. Garcés et al. 2002; Landès et al. 2010; Smets \& Evers 2014). Such studies demonstrate the advantage of an infrastructure that integrates independent middle atmospheric measurement techniques currently not assimilated in numerical weather prediction models (NWPs) and provides quantitative understanding of stratosphere-troposphere dynamical coupling useful for NWP applications (Le Pichon et al. 2015).

So far, microbarom studies have used qualitative comparisons between source models and received signals, with difficulties of interpretation associated with uncertainties in the measurements and in the propagation. Thanks to novel measurements from a stratospheric balloon fitted with microbarometers, Bowman \& Lees (2018) were able to verify quantitatively the predictions based on numerical ocean wave models and the microbarom source theory of Waxler et al. (2007) using measured sound spectra over the Southern Ocean. Their further interpretation 

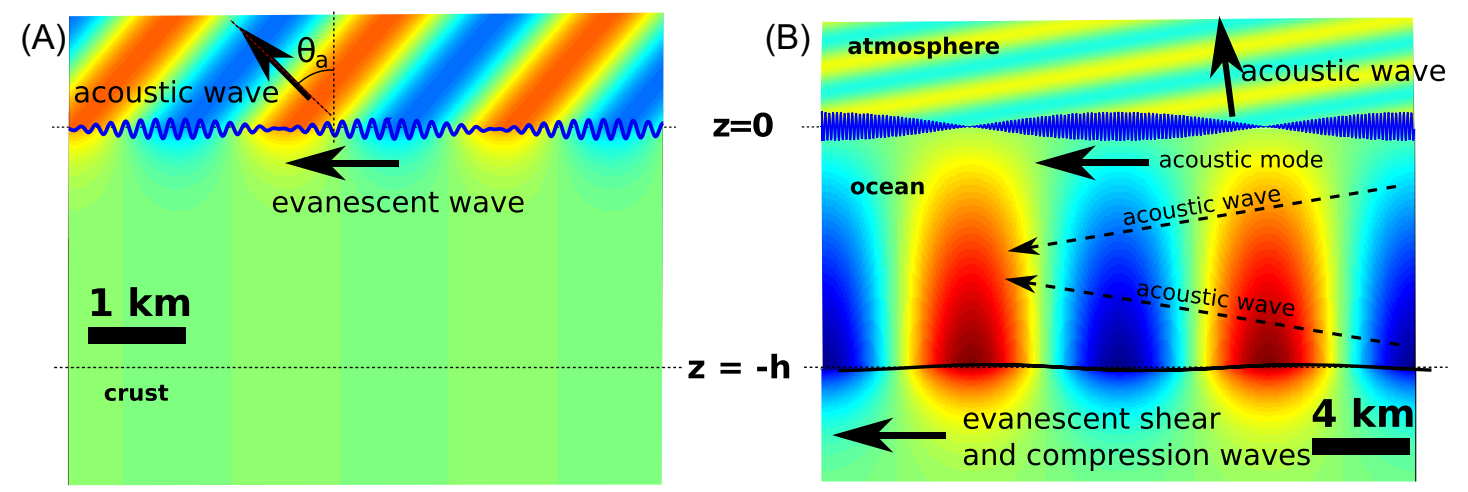

Figure 1. Example of vertical cross-section of the pressure pattern, in colours, radiated by a pair of interacting ocean wave trains of period around $10 \mathrm{~s}$. The sea surface elevation plotted as a blue line around $z=0$ is the sum of the elevations of the two wave trains and has a wave group structure. (a) The periods of the two wave trains are 10 and $9.66 \mathrm{~s}$ (b) 10 and $9.94 \mathrm{~s}$. Note that the vertical displacement of the sea surface is strongly exaggerated in order to make waves visible. A realistic ocean wave field includes many wave trains and thus all possible pairs of interactions radiating acoustic waves in all directions $\theta_{a}$. As the two periods of the wave train get closer, from (a) to (b), the lengths of the groups get larger and the angle $\theta_{a}$ becomes smaller. This paper focuses on the radiated power as a function of $\theta_{a}$.
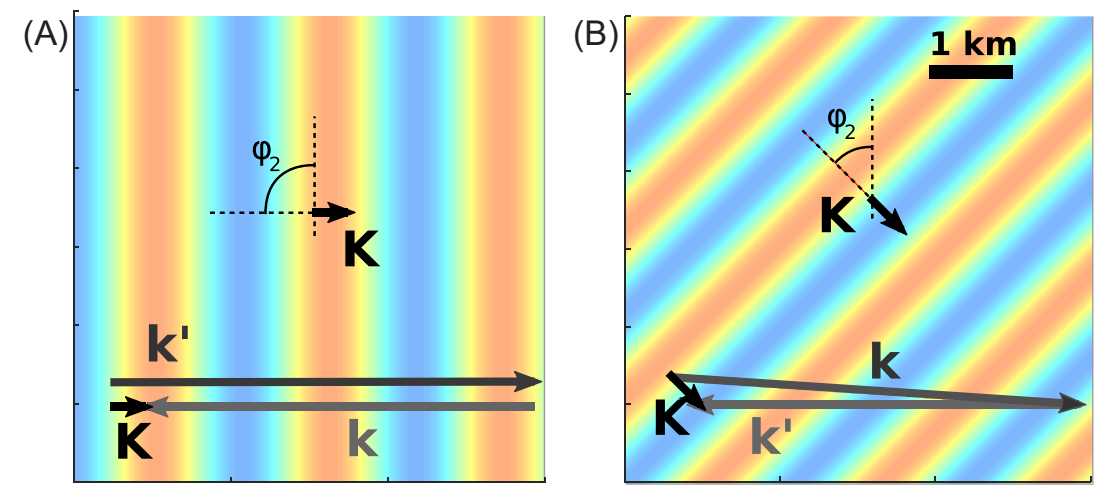

Figure 2. Example of horizontal pressure patterns, in colours, radiated by a pair of interacting ocean wave trains of periods 10 and $9.66 \mathrm{~s}$ with (a) opposing directions and (b) not exactly opposing directions. For acoustic waves, the maximum magnitude $K$ of the wavenumber vector $\mathbf{K}$ corresponds to horizontal propagation with $K=\Omega / \alpha_{a}$ and can be in any azimuth $\varphi_{2}$ depending on the exact ocean wave wavenumbers $\mathbf{k}$ and $\mathbf{k}^{\prime}$. Modes with larger values of $K$ decay exponentially over the vertical and are not relevant for microbaroms measured on land.

of microbaroms as a major heat source for the thermosphere, well above their measurement altitude, relies on the monopolar radiation pattern predicted by Waxler \& Gilbert (2006).

Following the work of Longuet-Higgins (1950) on microseisms, a first theory of microbarom generation was proposed by Posmentier (1967), with the atmospheric motion coming from the continuity of the velocity field at the air-sea interface, and no feedback of the atmosphere on the pressure field in the ocean. A more complete theory for random waves consistent with the microseism generation theory of Hasselmann (1963) and an accurate treatment of the air-sea boundary condition is given by Brekhovskikh et al. (1973), leading to significant differences for near-horizontal propagation. However, that work only considered an ocean of infinite depth. The effect of the ocean depth, with the amplification of particular frequencies corresponding to an 'organ pipe resonance' of the water column, was later considered by Waxler et al. (2007), extending the work done by Waxler \& Gilbert (2006). The major difference between that work and the earlier analysis of Brekhovskikh et al. (1973) is the monopole radiation pattern that, as we show here, comes from an assumption on the coherence of the source over only very short scales whereas Brekhovskikh et al. (1973) did not introduce this assumption. A unified theory is thus necessary for further quantitative analysis of microbarom records and the analysis of their impact in regions where no measurement is available, such as the thermosphere.

Given that microbaroms and microseisms are related (Donn \& Naini 1973), it is interesting to discuss microseisms for which more quantitative analyses are available. Microseisms have their most energetic sources associated to severe ocean storms but not necessarily colocated with the storm due to the propagation of ocean waves as swells (Obrebski et al. 2012). In particular, measurements at seismic stations near coasts can be dominated by the interaction of storm waves with their reflection from the coast (Bromirski et al. 1999; Ardhuin et al. 2011). In general, the sources at a frequency $f_{s}$ correspond to the interaction of waves with similar frequencies $f=f_{s} / 2$ and nearly opposite directions, as illustrated in Fig. 1 and Fig. 2 .

The generation of acoustic and seismic modes in the ocean and solid Earth with horizontal propagation speeds that exceed $1500 \mathrm{~m} \mathrm{~s}^{-1}$, much more than the typical ocean wave phase speeds around $15 \mathrm{~m} \mathrm{~s}^{-1}$, was explained quantitatively by Hasselmann (1963) as the result of an interference of pairs of ocean wave trains of wavenumbers $\mathbf{k}$ and $\mathbf{k}^{\prime}$, giving seismic or acoustic waves at the wavenumber $\mathbf{K}=\mathbf{k}+$ 
$\mathbf{k}^{\prime}$. The microseism generation theory is one particular example of the general theory of wave-wave interactions developed by Hasselmann (1966). The horizontal radiation pattern of a single pair of ocean waves gives a single sinusoidal pressure field propagating in the direction $\varphi_{2}$ of the wavenumber vector $\mathbf{K}$. For microseisms $K=|\mathbf{K}|$ is generally much smaller than the width of the ocean wave spectrum, so that the combination of all pairs of ocean waves gives an isotropic source.

In the case of microbaroms of frequency $0.2 \mathrm{~Hz}$, the sound speed in the air is only 20 times the phase speed of the ocean waves, so that $K$ is comparable to the ocean wave spectrum width, and there may be a preferential radiation in some directions $\varphi_{2}$. Applications and further analysis of microbaroms require a knowledge of the source magnitude and variability. In recent work, Waxler et al. (2007) investigated the influence of the water depth on the source magnitude, similar to what is found for microseisms. The conclusion of this paper is that the effect of water depth depends on the angle $\theta_{a}$ of the sound propagation relative to the vertical direction, and that the pressure field of microbaroms over the ocean generally contains a wide range of angles. Indeed, the coupling of the ocean and atmosphere strongly depends on $\theta_{a}$, as demonstrated by Brekhovskikh et al. (1973), but neglected by Waxler \& Gilbert (2006).

This importance of $\theta_{a}$ is now well known for microseisms and is easy to understand in relation to the physical properties of the solid Earth, ocean and atmosphere. A usual approximation of the propagating medium is a stack of uniform horizontal layers $l$ characterized by different velocities of propagation $\alpha_{l}$ and $\beta_{l}$ for compression and shear waves. Close to the source, microseisms are dominated by Rayleigh waves that correspond to relatively slow components, with horizontal propagation speeds between the sound speed in water $\alpha_{w}$, and the shear wave speed in the crust $\beta_{c}$. These Rayleigh modes combine motions that decay exponentially with depth in the solid Earth, with propagating acoustic waves in the ocean, and their acoustic propagation angles in the water $\theta_{w}$ are larger than $\arcsin \left(\alpha_{w} / \beta_{c}\right) \simeq 30^{\circ}$ (Ardhuin et al. 2019). For very large distances, seismic body waves may dominate the signal because of their weaker attenuation with distance, and these are due to the ocean acoustic noise that is more nearly vertical, allowing propagation in the crust, with $\theta_{w}<\arcsin \left(\alpha_{w} / \alpha_{c}\right) \simeq 16^{\circ}$. The water depth effect is clearly different for Rayleigh and body waves, as predicted by Ardhuin \& Herbers (2013) and Gualtieri et al. (2014), and demonstrated by Obrebski et al. (2013) and Meschede et al. (2017).

Now looking at microbaroms, we expect similar dependencies on $\theta_{a}$ because different components of the ocean wave forcing field with wavenumbers $\mathbf{K}=\mathbf{k}+\mathbf{k}^{\prime}$ give acoustic modes that have different apparent horizontal speeds $C=2 \pi f_{s} /|\mathbf{K}|$. Speed faster than the compression speed in the crust $C>\alpha_{c}$ leads to the generation of compression $P$ waves in the solid Earth, corresponding to nearly vertical propagation in the ocean layer and even more vertical propagation in the atmosphere, given by Snell's law. The limit $C \rightarrow \infty$ corresponding to vertical propagation and $|\mathbf{K}|=0$, which are the exactly standing waves of Longuet-Higgins. At the other extreme, $C<\alpha_{w}$ gives evanescent waves in the water layer that correspond to acoustic-gravity (AG) modes that dominate the pressure field measured in the top $300 \mathrm{~m}$ of the ocean, as observed by Cox \& Jacobs (1989) and Ardhuin et al. (2013). For these AG modes, we expect no influence of water depths larger than $300 \mathrm{~m}$ on the pressure at the ocean surface. These AG waves are coupled to atmospheric acoustic waves that have propagation angles larger than $\theta_{a 0}=\arcsin \alpha_{a} / \alpha_{w} \simeq 12^{\circ}$.

The difference in water depth effects between body waves, Rayleigh waves and AG modes should influence the amplitude of acoustic waves radiated in the atmosphere, and the amplitude of microbaroms should strongly depend on the direction of propagation $\theta_{a}$ relative to the vertical. The decomposition of the ocean wave forcing in different horizontal wavenumbers $\mathbf{K}=K$ ( $\cos \varphi_{2}$, $\sin \varphi_{2}$ ) allows to $\operatorname{consider}$ separately the different acoustic wave components and how they may contribute to different acoustic modes. Each $K$ corresponds to a propagation angle such that $\sin \theta_{l}=K \alpha_{l} /\left(2 \pi f_{s}\right)$, where $\alpha_{l}$ is the sound speed in the layer $l$, which is related to Snell's law.

Because the ocean wave spectra are relatively broad, they contain a wide range of pairs $\mathbf{k}$ and $\mathbf{k}^{\prime}$ so that all possible $\mathbf{K}$ are excited simultaneously. For microseisms, this produces a spectrum of the excitation that is white in wavenumber, and thus equivalent to a point force. In the case of microbaroms, the conversion from wave motion to acoustic pressure is a function of the wavenumber $K$, first given by Brekhovskikh et al. (1973), which determines the radiation pattern in the atmosphere.

Hence the previous works either lack the possible important effect of finite depth in the ocean or important aspects of the radiation pattern in the atmosphere. In order to progress towards a quantitative understanding of microbarom signals, it is thus necessary to have both effects in the same theory, and this is the objective of this paper. For the sake of simplicity, we only consider the case of a homogeneous atmosphere and extend the theory of Brekhovskikh et al. (1973) to take into account a finite water depth. The theoretical formulation and the main results are given in Section 2, with details of the derivation in the Supporting Information. These results are interpreted in Section 3 and conclusions follow in Section 4.

\section{A GENERAL THEORY OF MICROBAROM SOURCES}

In order to facilitate the translation between the different papers we have listed in Table 1 the correspondence of the main symbols used. We have also included Longuet-Higgins (1950) because it treats almost the same physical problem, with a focus on the water layer, and the same decomposition in particular and homogeneous solutions of the forced wave equation.

As detailed in the Supporting Information, which follows the method of Brekhovskikh et al. (1973), the basis of microseism and microbaroms generation theory is the coupling of motions in different layers, with a forcing coming from nonlinear ocean wave effects, in which the nonlinearity is necessary to allow the generation of waves with long wavelengths $2 \pi /\left|\mathbf{k}+\mathbf{k}^{\prime}\right|$ from the interference of shorter ocean 
Table 1. Notations used in different papers: LH50 stands for Longuet-Higgins (1950), BGKN73 stands for Brekhovskikh et al. (1973), WG06 stands for Waxler \& Gilbert (2006) and AH13 stands for Ardhuin \& Herbers (2013).

\begin{tabular}{lccccc}
\hline Quantity & This paper & LH50 & BGKN73 & WG06 & AH13 \\
\hline Vertical coordinate & $z$ & $-z$ & $z$ & $z$ & $z$ \\
Angle relative to vertical & $\theta_{a}$ or $\theta_{w}$ & - & $\theta_{1}$ or $\theta_{2}$ & - & - \\
Surface elevation & $\zeta$ & $\zeta$ & $\zeta$ & $\xi$ & $\zeta$ \\
Azimuth of spectrum & $\varphi$ & $\theta$ & $\varphi$ & $\theta$ & $\theta$ \\
Azimuth of acoustic signal & $\varphi_{2}$ & - & $\varphi_{a}$ & - & - \\
Velocity potential & $\phi$ & $-\phi$ & $-\varphi$ & $\phi$ & $\phi$ \\
Layer index & $l$ & - & $j$ & $\sigma$ & - \\
Sound speed & $\alpha_{l}$ & $c$ & $c_{j}$ & $c_{\sigma}$ & $\alpha$ \\
Density ratio & $m$ & - & $m$ & - & - \\
Horizontal wavenumber & $\mathbf{K}$ & - & $\mathbf{q}$ & - & $\mathbf{K}$ \\
Radian frequency & $\Omega$ & - & $\Omega$ & - & $2 \pi f_{s}$ \\
Horizontal wavenumbers & $\mathbf{k}, \mathbf{k}^{\prime}$ & $(-u k,-v k)$ & $\varkappa, \varkappa_{1}$ & $\mathbf{k}, \mathbf{q}$ & $\mathbf{k}, \mathbf{k}$ \\
Radian frequencies & $\sigma, \sigma$ & $\sigma$ & $\omega(\varkappa), \omega(\varkappa \mathbf{r})$ & $\omega(\mathbf{k}), \omega(\mathbf{q})$ & $\sigma, \sigma^{\prime}$ \\
Pressure & $p$ & $p$ & $\rho \mathcal{P}$ & $p$ & $p$ \\
Vertical wavenumbers & $v, \mu$ &,$- \alpha$ & $\lambda_{1}, \lambda_{2}$ & - & $l_{a}, l$ \\
Upward amplification & $g / 2 \alpha_{l}$ & $\gamma$ & - & - & - \\
\hline
\end{tabular}

Table 2. Summary of differences between models, with $R_{a}$ defined in eq. (29).

\begin{tabular}{lccccc}
\hline Model & Depends on $\theta_{a}$ & Depth & Compressible & Gravity & $R_{a}$ \\
\hline BGKN73 & $\sqrt{ }$ & $\infty$ & $\sqrt{ }$ & $\sqrt{ }$ & eq. (30) \\
AH13 & $\sqrt{ }$ & $\infty$ & $\times$ & $\times$ & eq. (31) \\
WG06 & $\times$ & $\infty$ & $\sqrt{ }$ & $\times$ & eq. (32) \\
W07 & $\times$ & any & $\sqrt{ }$ & $\times$ & eq. (35) \\
this paper & $\sqrt{ }$ & any & $\sqrt{ }$ & $\sqrt{ }$ & eq. (41) \\
\hline
\end{tabular}

waves with wavelengths $2 \pi /|\mathbf{k}|$ and $2 \pi /|\mathbf{k}|^{\prime}$. The velocity potential $\phi$ in layer $l$ is solution of a wave equation (Brekhovskikh et al. 1973),

$\frac{\partial^{2} \phi}{\partial t^{2}}-\alpha_{l}^{2} \nabla^{2} \phi=-\frac{\partial}{\partial t}(\nabla \phi)^{2}-g \frac{\partial \phi}{\partial z}$,

where $\nabla^{2}$ is the 3-D Laplace operator. The two terms on the right-hand side can be neglected in the water layer (Longuet-Higgins 1950), but are generally significant in the air. The first term corresponds to the effect of compressibility. It adds one particular solution $\phi_{p}$ that is zero away from the boundary but modifies the homogeneous solution via the boundary condition at the air-sea interface. The second term is the effect of gravity, which gives a weak additional $\exp \left(g z / 2 \alpha_{l}^{2}\right)$ vertical decay, with a half-decay distance of $15 \mathrm{~km}$ in the atmosphere and $300 \mathrm{~km}$ in the ocean. That second effect was considered by Brekhovskikh et al. (1973) but neglected in Waxler \& Gilbert (2006).

Neglecting these two terms for the water layer, solutions that are periodic in time and space take the following homogeneous form, with $\Omega=2 \pi f_{s}$ the radian frequency and $\kappa_{a}=v_{+}$and $\kappa_{w}=\mu_{-}$the vertical wavenumbers (going upwards in the air and downwards in the water)

$$
\begin{aligned}
\phi & =\sum_{\mathbf{k}} \Phi_{l} \exp \left[\mathrm{i}\left(\mathbf{K} \cdot \mathbf{x}+\kappa_{l} z-\Omega t\right)\right]+\text { c.c. } \\
& =\sum_{\mathbf{k}, s} \Phi_{l} \exp \left[\mathrm{i}\left(\mathbf{K} \cdot \mathbf{x}+\kappa_{l} z-s \Omega t\right)\right]
\end{aligned}
$$

where c.c. stands for the complex conjugate and $s=1$ or $s=-1$ is a sign index. Neglecting the right-hand side of (1), one gets

$\kappa_{l}^{2}+K^{2}=\Omega^{2} / \alpha_{l}^{2}$.

With $N$ the number of unknown potential amplitudes $\left(\Phi_{i}\right)_{1 \leq i \leq N}$, there are $N$ continuity conditions for stresses and displacements at the layer interfaces, linking the $N$ amplitudes of velocity potentials.

All the variables, the pressure $p$, the density $\rho$, the velocity potential $\phi$ and the sea surface elevation $\zeta$ are expanded in powers of $\varepsilon=a k$ that is the product of a typical ocean wave wavenumber $k$ and surface elevation amplitude $a$,

$p=p_{0}+p_{1}+p_{2}+\ldots$

$\rho=\rho_{0}+\rho_{1}+\rho_{2}+\ldots$

$\phi=\phi_{0}+\phi_{1}+\phi_{2}+\ldots$

$\zeta=\zeta_{0}+\zeta_{1}+\zeta_{2}+\ldots$ 
In addition to the wave slope $\varepsilon$, two other small parameters are defined, the ratio between the air and water densities $m=\rho_{a} / \rho_{w}$ and $\delta_{l}$ $=\sigma / k \alpha_{l}$ the ratio between the speed of surface waves and the speed of sound in the air or water.

Collecting the terms of same order, we obtain at each order a system of $N$ equations for $N$ unknowns with a detailed derivation in Supporting Information. At order $\varepsilon^{0}$, the solution is the hydrostatic equilibrium of pressure and gravity. The first-order solution corresponds to Airy waves, which are linear gravity waves, with negligible $O\left(\delta_{w}^{2}\right)$ and $O(m)$ corrections due to the presence of air and the compressibility of air and water, as given by Brekhovskikh et al. (1973, eqs 11 and 12). See also the Supporting Information. Namely the surface elevation is given by Hasselmann (1962),

$\zeta_{1}(\mathbf{x}, t)=\sum_{\mathbf{k}, s} Z_{1, \mathbf{k}}^{s} \exp [\mathrm{i}(\mathbf{k} \cdot \mathbf{x}-s \sigma t)]$

where $Z_{1, \mathbf{k}}^{s}$ is the amplitude of the first-order sea surface elevation for wavenumber $\mathbf{k}$ and propagation direction $s$ and $s= \pm 1$ is a sign index that gives the direction of propagation relative the direction of the wave vector $\mathbf{k}$. The velocity potential in the water is given by

$\phi_{w}(\mathbf{x}, z, t)=\sum_{\mathbf{k}, s} \Phi_{1, \mathbf{k}}^{s} \frac{\cosh (k z+k h)}{\cosh (k h)} \exp [\mathrm{i}(\mathbf{k} \cdot \mathbf{x}-s \sigma t)]$

with

$\Phi_{1, \mathbf{k}}^{s}=\frac{g}{\mathrm{i} \sigma} Z_{1, \mathbf{k}}^{s}$.

In the air, the effects of gravity and compressibility (i.e. the right-hand side terms in eq. 1) are less negligible, and we have

$\phi_{a}(\mathbf{x}, z, t)=\sum_{\mathbf{k}, s} \frac{k}{k_{a}} \Phi_{1, \mathbf{k}}^{s} \exp \left[-k_{a} z+\mathrm{i}(\mathbf{k} \cdot \mathbf{x}-s \sigma t)\right]$

with eq. (12) in Brekhovskikh et al. (1973),

$k_{a}=k\left(\sqrt{1-\delta_{a}^{2}+\delta_{a}^{4} / 2}-\delta_{a}^{2} / 2\right) \simeq k\left(1-\delta_{a}^{2}\right)$

where $\delta_{a}=\sqrt{g / k} / \alpha_{a}$. We note that half of this correction to $k_{a}$ comes from the $\delta_{a}^{2} / 2$ that is due to gravity, and the other half comes from the air compressibility.

Finally, in the $\varepsilon^{2}$ system, the wave spectrum acts as a forcing, coming through either the particular solutions that satisfy the wave equation with the right-hand side or from the boundary conditions between the different layers. In other words, the wave forcing $\Lambda=\left(\Lambda_{i}\right)_{1} \leq i \leq N$ is a vector on the right-hand side of a matrix equation

$\mathbf{M} \Phi=\Lambda$.

The only differences between all the theories discussed here are in the approximations of the boundary conditions between ocean and atmosphere and ocean and solid Earth. Mathematically, different terms are neglected in the coefficients of the matrix $\mathbf{M}$ or in the forcing vector $\boldsymbol{\Lambda}$, as detailed below.

Further extensions to multiple layers in the atmosphere and solid Earth give rise to different horizontally propagating modes, which correspond to zeros of the determinant of $\mathbf{M}$, for which a growth rate of the energy can be computed as done for seismic Rayleigh waves by Hasselmann (1963). The size of the matrix M grows by two lines and columns for each extra fluid layer, for which the two unknowns are one upward and one downward propagating potential amplitudes. For a solid layer there are four unknowns due to the presence of both compression and shear motions (see Hasselmann 1963, eq. 1.4). The important difference with Hasselmann (1963) is that $\Lambda_{i}$ was non-zero only for the sea surface pressure continuity equation in Hasselmann's case, whereas in our case we will consider forcing in both the pressure and velocity equations. We also note that for finite water depth there is also a forcing term in the boundary condition for the ocean bottom pressure coming into $\Lambda_{3}$ (Ardhuin \& Herbers 2013).

\subsection{Existing solutions}

\subsubsection{Case of infinite water depth-Brekhovskikh et al. (1973)}

Brekhovskikh et al. (1973) considered only two layers, air and water, that are half-spaces. As a result, for each frequency $\Omega$ and wavenumber vector $\mathbf{K}$, there are only two unknowns, one amplitude $A$ for the velocity potential of upgoing acoustic waves in the atmosphere, and one amplitude $W$ for downgoing acoustic waves or evanescent modes in the ocean. The approximation $m=\rho_{a} / \rho_{w} \ll 1$ removes the feedback of the atmospheric pressure on the air pressure, so that the atmosphere is only driven by the continuity of vertical velocities at the interface.

The coupling of air and water layers at $z=0$ by the continuity of pressure and velocity gives a $2 \times 2$ matrix $\mathbf{M}$, with one line for the continuity of vertical velocity $w=\partial \phi / \partial z$ and the other for the continuity of pressure $p$,

$M_{1,1} A+M_{1,2} W=\Lambda_{1}$ 
$M_{2,1} A+M_{2,2} W=\Lambda_{2}$.

Following Brekhovskikh et al. (1973), we introduce the small parameters

$\delta_{a}^{2}=g /\left(k \alpha_{a}^{2}\right)$

$m=\rho_{a} / \rho_{w}$

$n=\alpha_{a} / \alpha_{w}$

and we note that $|K / k|<2 \delta_{a}$. We now keep only the lowest order terms in $\delta_{a}$ and $m$, giving the following matrix coefficients and right-hand side (see the Supporting Information for details),

$M_{1,1}=v_{+}=\mathrm{i} \frac{\Omega}{\alpha_{a}} \cos \theta_{a}+\frac{k}{2} \delta_{a}^{2}$

$M_{1,2}=-\mu_{-}=2 k \delta_{a} l-\frac{\delta_{a}^{2}}{2} k n^{2}$

$M_{2,1}=-m \Omega^{2}-g i \frac{\Omega}{\alpha_{a}} \cos \theta_{a}$

$M_{2,2}=\Omega^{2}$

$\Lambda_{1}=$ is $\Omega k \delta_{a}^{2}\left(2-2 \sin ^{2} \theta_{a}\left(1-\frac{1}{2} \cos ^{2}\left(\varphi_{2}-\varphi\right)\right)+n^{2}\right)$

$\Lambda_{2}=\frac{\text { is } \Omega}{\rho_{w}} p_{\text {surf }}^{s}(\mathbf{K}, \Omega)+o\left(\delta_{a}^{2}\right)$

where we have defined

$l=\left(\sin ^{2} \theta_{a}-n^{2}\right)^{1 / 2}=n \cos \theta_{w} \quad$ if $\quad \theta_{a}<\theta_{a 0}$.

Following Hasselmann (1963), we define the amplitude of the equivalent surface pressure induced at second order by the wave motion. Assuming that $k h \gg 1$, we take the following definition:

$p_{\text {surf }}^{s}(\mathbf{K}, \Omega)=-2 \rho_{w} \sum_{\mathbf{k}+\mathbf{k}^{\prime}=\mathbf{K}, \sigma+\sigma^{\prime}=\Omega} \sigma \sigma^{\prime} Z_{1, \mathbf{k}}^{s} Z_{1, \mathbf{k}^{\prime}}^{s}$

In the following we will write $p_{\text {surf }}^{s}$ instead of $p_{\text {surf }}^{s}(\mathbf{K}, \Omega)$.

The solution of matrix eqs (14) and (15) is given by Cramer's rule,

$A=\frac{\Lambda_{1} M_{2,2}-\Lambda_{2} M_{1,2}}{\operatorname{det}(\mathbf{M})} \quad$ and $\quad W=\frac{\Lambda_{2} M_{1,1}-\Lambda_{1} M_{2,1}}{\operatorname{det}(\mathbf{M})}$.

Following details in Supporting Information Section S4.1, we find the amplitudes of the velocity potentials at the air-sea interface to be, for the water and air respectively,

$W \simeq \frac{\mathrm{i}}{\rho_{w}} \frac{1}{2 \sigma^{\prime}} p_{\text {surf }}^{s}$

$A \simeq \frac{R_{a}}{\rho_{w}} \frac{1}{2 \sigma^{\prime}} p_{\text {surf }}^{s}$

with

$R_{a}=\frac{l-2 \delta_{a}\left[1-\sin ^{2} \theta_{a}\left(1-\frac{1}{2} \cos ^{2}\left(\varphi_{2}-\varphi\right)\right)+\frac{5}{8} n^{2}\right]}{\cos \theta_{a}\left(1-\delta_{a} l / 2\right)-\mathrm{i}\left(\delta_{a} / 4+m l\right)}$.

This form of $R_{a}$ is identical to Brekhovskikh et al. (1973, eq. 22), except for the addition of one extra term $\cos \theta_{a} l \delta_{a} / 2$ in the denominator, and a change in the sign of the denominator term $\mathrm{ml}$. As shown in Fig. 3, these two terms have a negligible impact on the solution, except for $\theta_{a}>89.5^{\circ}$, with less than 1 per cent change in the total radiated acoustic power.

\subsubsection{A simplified case-Ardhuin \& Herbers (2013)}

The solution given by Ardhuin et al. (2013) corresponds to the simplified solution given by eq. (21) in Brekhovskikh et al. (1973), with $m$ and $\delta_{a}$ terms neglected, corresponding to an absence of feedback from the atmospheric pressure on the oceanic pressure, that is, $M_{1,1}=0$, 

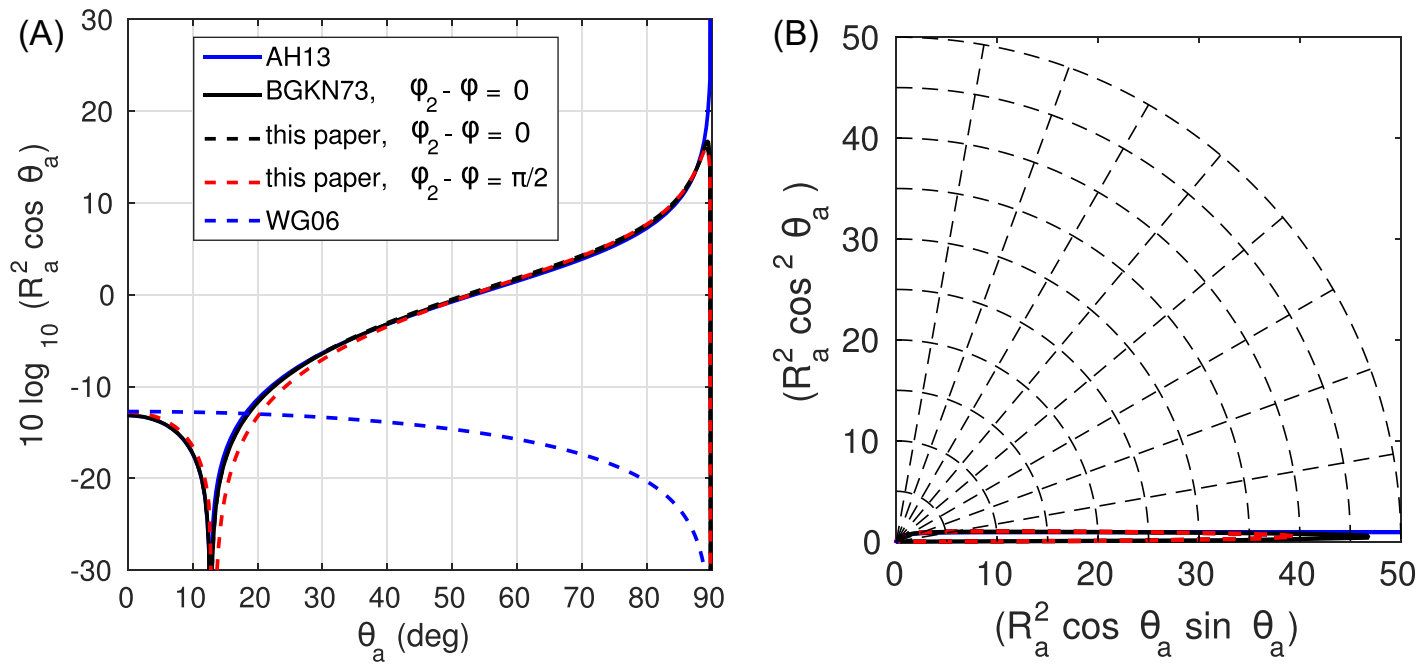

Figure 3. Patterns of acoustic pressure variance as a function of the elevation angle $\theta_{a}$ for an ocean wave period of $10 \mathrm{~s}$, given by the different theories without ocean bottom, in Cartesian (a) and polar (b) representation. Note that when the radiated power is considered, these patterns must be multiplied by $\sin \theta_{a}$ before integration over $\theta_{a}$, as given by eq. (52). In general, as given by eq. (30), the radiated power is also a function of the relative azimuth of the first ocean wave train $\varphi$ and the azimuth of the radiated acoustic power $\varphi_{2}$.

and neglecting the right-hand side of the acoustic wave equation in the air, that is, $\Lambda_{1}=0$, giving

$R_{a}=\frac{l}{\cos \theta_{a}}=\frac{\mathrm{i}\left(n^{2}-\sin ^{2} \theta_{a}\right)^{1 / 2}}{\cos \theta_{a}}$

where we recall that $l$ is imaginary for $\theta_{a}<\theta_{a 0}$.

This simplified solution corresponds to infinite water depth. It presents a singularity for horizontal acoustic propagation as $\cos \theta_{a}$ goes to zero. That singularity is removed when the feedback of the air on the water motion is taken into account.

\subsubsection{Theory by Waxler \& Gilbert (2006)}

Following Brekhovskikh et al. (1973), Waxler \& Gilbert (2006) showed that microbarom signals are due both to ocean radiation and to compression of the air by the surface motion, but Waxler \& Gilbert (2006) neglected the effect of gravity in the air. As detailed in the Supporting Information, accounting for gravity in the air changes their term $3 \delta_{a}^{2} / 2$ in their eq. (57) to $2 \delta_{a}$ in eq. (32). we also note a change of sign from -2 to 1.5 ,

$R_{a}^{\mathrm{WG} 06}=\mathrm{i} n+1.5 \delta_{a}$.

The particularity of the derivation by Waxler \& Gilbert (2006) is the fact that they neglect the phase shift in the Green's function within the source region. They justified that approximation by assuming that the coherence length scale in the acoustic source is small compared to the acoustic wavelength. Here we do not use such an approximation, as detailed in the Appendix, as the correlation function of the source is given by the pressure spectrum $p_{\text {surf }}^{s}(\mathbf{K}, \Omega)$ (our eq. 26) that overlaps with the acoustic wavelengths. The assumption in Waxler \& Gilbert (2006) comes between their eqs (50) and (51) and simplifies the expression of the radiation pattern in the atmosphere to a monopolar radiation pattern. It also reduces all expressions to their values for $\mathbf{K}=0$, corresponding to strictly opposing wave trains, so that the evanescent ocean motions that correspond to $\theta_{a}>\theta_{a 0}$ are not properly represented. Without this assumption, Brekhovskikh et al. (1973) found that the radiation pattern is very different from a monopole, with an overwhelming radiation at very grazing angles, as illustrated in Fig. 1(a).

\subsection{Generalization of Brekhovskikh et al. (1973) to a finite ocean depth}

The first discussion of water depth effects on microbarom sources is due to Waxler et al. (2007). That work extended the analysis by Waxler \& Gilbert (2006), limiting the water depth to $h$ and including an interface with a solid half-space below. The velocity potential in the water is now

$\phi_{w, 2}=\sum\left(W_{+} \mathrm{e}^{\mu_{+} z}+W_{-} \mathrm{e}^{\mu_{-} z}\right) \mathrm{e}^{\mathrm{i} \Theta}$,

with the complex wavenumber $\mu_{ \pm}=g /\left(2 \alpha_{w}\right) \pm \sqrt{K^{2}-\Omega^{2} / \alpha_{w}^{2}} \simeq \pm \mu= \pm \sqrt{K^{2}-\Omega^{2} / \alpha_{w}^{2}}$. 
Taking an acoustic reflection coefficient $r=\rho_{w} \alpha_{w} /\left(\rho_{s} \alpha_{s}\right)$, Waxler et al. (2007) found the reflection condition for the downgoing acoustic waves, with potential $\phi_{w}^{-}$at the ocean bottom

$W^{+}=\frac{1+r}{1-r} \mathrm{e}^{2 \mu h} W^{-}$.

This gives,

$R_{a}^{\mathrm{W} 07} \simeq \mathrm{in} \frac{r \cos \left(\Omega h / \alpha_{w}\right)+\mathrm{i} \sin \left(\Omega h / \alpha_{w}\right)}{\cos \left(\Omega h / \alpha_{w}\right)+\mathrm{i} r \sin \left(\Omega h / \alpha_{w}\right)}+1.5 \delta_{a}$.

In order to properly consider the effect of the propagation angles, we can go back to the derivation of Brekhovskikh et al. (1973), now including an upgoing acoustic wave in the water layer. For oblique incidence we have to consider the contribution of compression waves and shear waves in the crust with velocities $\alpha_{s}$ and $\beta$. Defining the vertical wavenumbers in the crust

$\chi_{p}=\sqrt{K^{2}-\frac{\Omega^{2}}{\alpha_{s}^{2}}}$ and $\chi_{s}=\sqrt{K^{2}-\frac{\Omega^{2}}{\beta^{2}}}$,

the reflection at the bottom generalizes to

$r \simeq \frac{\Omega^{4} \rho_{w} \chi_{p}}{\rho_{s} \mu\left[\left(\Omega^{2}-2 K^{2} \beta^{2}\right)^{2}-4 \beta^{4} K^{2} \chi_{p} \chi_{s}\right]}$.

This is obtained by eliminating the potentials of the compression and shear motions in the crust, using the continuity of velocity and a zero tangential stress (see also Ardhuin \& Herbers 2013; Gualtieri et al. 2014).

When only the dominant terms are kept, we find

$R_{a}=\frac{l}{\cos \theta_{a}} \frac{R}{Q}$

with

$R=\sinh (\mu h)+r \cosh (\mu h)$,

$Q=\cosh (\mu h)+r \sinh (\mu h)$.

In the case $\theta_{a}=0$, eq. (38) corresponds to the first term of eq. (35).

When going to first order in $\delta_{a}$ and $m$, the problem can be simplified by neglecting the effect of gravity in the water layer, which contributed to the $n^{2}$ term in the numerator of eq. (30). This gives $-\mu_{-}=\mu_{+}=\mu$. It is then more simple to eliminate the amplitude $W^{-}$ by using the bottom boundary condition on the vertical velocity. This amounts to replacing $-\mu_{-}$by $-2 \mu R \mathrm{e}^{-\mu h} /(1+r)$ in $M_{1,2}$ and $\Omega^{2}$ by $2 Q \Omega^{2} \mathrm{e}^{-\mu h} /(1+r)$ in $M_{2,2}$, giving,

$R_{a}=\frac{l R-2 \delta_{a} Q\left[1-\sin ^{2} \theta_{a}\left(1-\frac{1}{2} \cos ^{2}\left(\varphi_{2}-\varphi\right)\right)\right]}{\cos \theta_{a}\left(Q+R \delta_{a} l / 2\right)-\mathrm{i}\left(Q \delta_{a} / 4+R m l\right)}$.

Fig. 4. shows the variations of the velocity potential amplification from the water to the air, $R_{a}$, depending on $\theta_{a}$ and the depth $h$. In the case $\theta_{a}=0$, Fig. 5. compares the new formulation of $R_{a}$ to the one from Waxler et al. 2007 as a function of the ratio of the water depth to the acoustic wavelength. The models discussed in sections 2.1 and 2.2 are summarized in Table 2.

\subsection{Radiated acoustic power as a function of elevation and azimuth}

We introduce the spectral density of the homogeneous (propagating) pressure field at $z=0$, in the three spectral dimensions $\left(K_{x}, K_{y}, f_{s}\right)$ using the Fourier amplitude of $p_{a, 2}$ at $z=0$, obtained from the average over realizations of the sea state, represented by angular brackets, of the pressure amplitude squared,

$F_{p, 2 h}\left(\mathbf{K}, f_{s}\right)=2 \lim _{\Delta K_{x} \rightarrow 0, \Delta K_{y} \rightarrow 0, d f_{s} \rightarrow 0} \frac{\left\langle\left|P_{2, h}^{+}\right|^{2}\left(\mathbf{K}, f_{s}\right)\right\rangle}{\Delta K_{x} \Delta K_{x} \Delta f_{s}}$.

Given the dispersion relation of ocean surface gravity waves in deep water, the Jacobian of the transformation from $\left(k_{x}, k_{y}\right)$ to $(f, \varphi)$ is $2 \pi k /(\partial \sigma / \partial k)=4 \pi \sigma^{3} / g^{2}$.

We now define the ocean wave spectrum as

$$
\begin{aligned}
E(f, \varphi) & =\frac{4 \pi \sigma^{3}}{g^{2}} E\left(k_{x}, k_{y}\right)=2 \lim _{\Delta k_{x} \rightarrow 0, \Delta k_{y} \rightarrow 0} \frac{\left|Z_{\mathbf{k}}^{+}\right|^{2}}{\Delta K_{x} \Delta K_{y}} \frac{4 \pi \sigma^{3}}{g^{2}} \\
& =2 \lim _{\Delta f \rightarrow 0, \Delta \varphi \rightarrow 0} \frac{\left|Z_{\mathbf{k}}^{+}\right|^{2}}{\Delta f \Delta \varphi} .
\end{aligned}
$$


We use eq. (26) and replace the amplitude $P_{2, h}^{+}$by $\mathrm{i}\left(\sigma+\sigma^{\prime}\right) \rho_{a} A$, namely,

$P_{2, h}^{+}=\frac{\mathrm{i} \rho_{a}\left(\sigma+\sigma^{\prime}\right)}{\rho_{w} 2 \sigma^{\prime}} R_{a} p_{\text {surf }}^{+}$.

This gives,

$$
\begin{aligned}
& 2\left|P_{2, h}^{+}\right|^{2}=2 \rho_{a}^{2}\left|R_{a}\right|^{2} \sigma^{2}\left(\sigma+\sigma^{\prime}\right)^{2}\left|\sum_{\mathbf{k}+\mathbf{k}^{\prime}=\mathbf{K}, \sigma+\sigma^{\prime}=\Omega} Z_{1, \mathbf{k}} Z_{1, \mathbf{k}^{\prime}}\right|^{2} \\
& =\sigma^{2}\left(\sigma+\sigma^{\prime}\right)^{2} \rho_{a}^{2}\left|R_{a}\right|^{2} \sum_{\mathbf{k}+\mathbf{k}^{\prime}=\mathbf{K}, \sigma+\sigma^{\prime}=\Omega} 2\left|Z_{1, \mathbf{k}}^{+}\right|^{2} 2\left|Z_{1, \mathbf{k}^{\prime}}^{+}\right|^{2},
\end{aligned}
$$

where the last equality is obtained by considering that each pair of wavenumbers $\left(\mathbf{k}_{1}, \mathbf{k}_{2}\right)$ is counted twice, a first time when $\mathbf{k}=\mathbf{k}_{1}$ and $\mathbf{k}$ $=\mathbf{k}_{2}$ and a second time when $\mathbf{k}=\mathbf{k}_{2}$ and $\mathbf{k}^{\prime}=\mathbf{k}_{1}$. This is well understood when considering the simplest form with the ocean wave field consisting of only two cosine waves (see Supporting Information eqs S124-S128).

Taking the limit to continuous sums and using a change of variable from $\left(k_{x}, k_{y}, k_{x}^{\prime}, k_{y}^{\prime}\right)$ to $\left(f_{s}, \varphi, K_{x}, K_{y}\right)$, with $K_{x}=k_{x}+k_{x}^{\prime}$, $K_{y}=k_{y}+k_{y}^{\prime}$ and $f_{s}=\left(\sqrt{g k}+\sqrt{g k^{\prime}}\right) /(2 \pi)$ the Jacobian of the coordinate transform is

$$
\begin{aligned}
\operatorname{det}\left(\frac{\partial f_{s} \partial \varphi \partial K_{x} \partial K_{y}}{\partial k_{x} \partial k_{y} \partial k_{x}^{\prime} \partial k_{y}^{\prime}}\right) & =\left|\begin{array}{cccc}
g \cos \varphi /(4 \pi \sigma) & -\sin \varphi / k & 1 & 0 \\
g \sin \varphi /(4 \pi \sigma) & \cos \varphi / k & 0 & 1 \\
g \cos \varphi^{\prime} /\left(4 \pi \sigma^{\prime}\right) & 0 & 1 & 0 \\
g \sin \varphi^{\prime} /\left(4 \pi \sigma^{\prime}\right) & 0 & 0 & 1
\end{array}\right| \\
& =\frac{g^{2}}{4 \pi \sigma^{3} \sigma^{\prime}}\left[\sigma^{\prime}-\sigma \cos \left(\varphi-\varphi^{\prime}\right)\right],
\end{aligned}
$$

and gives

$$
\begin{aligned}
\int F_{p, 2 h}\left(\mathbf{K}, f_{s}\right) \mathrm{d} K_{x} \mathrm{~d} K_{y} \mathrm{~d} f_{s} & =\rho_{a}^{2} \int \sigma^{2}\left(\sigma+\sigma^{\prime}\right)^{2}\left|R_{a}\right|^{2} E\left(k_{x}, k_{y}\right) E\left(k_{x}, k_{y}\right) \mathrm{d} k_{x} \mathrm{~d} k_{y} \mathrm{~d} k_{x}^{\prime} \mathrm{d} k_{y}^{\prime} \\
& =\rho_{a}^{2} \int \sigma^{2}\left(\sigma+\sigma^{\prime}\right)^{2}\left|R_{a}\right|^{2} \frac{E\left(k_{x}, k_{y}\right) E\left(k_{x}^{\prime}, k_{y}^{\prime}\right) 4 \pi \sigma^{3} \sigma^{\prime}}{g^{2}\left[\sigma^{\prime}-\sigma \cos \left(\varphi-\varphi^{\prime}\right)\right]} \mathrm{d} f_{s} \mathrm{~d} \varphi \mathrm{d} K_{x} \mathrm{~d} K_{y} .
\end{aligned}
$$

Now we use the uniqueness of the Fourier transform to identify the spectral density in the left- and right-hand sides, and using eq. (43) gives

$F_{p, 2 h}\left(\mathbf{K}, f_{s}\right)=\frac{1}{2} \rho_{a}^{2} g^{2} f_{s} \int_{0}^{2 \pi} \frac{\sigma^{2}\left(\sigma+\sigma^{\prime}\right)}{\sigma^{\prime 2}}\left|R_{a}\right|^{2} \frac{E(f, \varphi) E\left(f^{\prime}, \varphi^{\prime}\right)}{\sigma^{\prime}-\sigma \cos \left(\varphi-\varphi^{\prime}\right)} \mathrm{d} \varphi$.

We note that the form of the acoustic power given by eq. (48) is generally a function of the direction $\varphi_{2}$ of the horizontal wave vector $\mathbf{K}$ of the acoustic waves.

In the limit $\delta_{a} \ll 1$, this simplifies to a horizontally isotropic form

$F_{p, 2 h}\left(\mathbf{K}, f_{s}\right) \simeq \frac{1}{2} \rho_{a}^{2} g^{2} f_{s}\left|R_{a}\right|^{2} \int_{0}^{2 \pi} E(f, \varphi) E(f, \varphi+\pi) \mathrm{d} \varphi$.

This last expression, with $\left|R_{a}\right|^{2} \simeq\left|\left(\sin ^{2} \theta_{a}-n^{2}\right)\right| / \cos ^{2} \theta_{a}$, is the one used by Ardhuin \& Herbers (2013).

The pressure spectrum can be re-written as a directional spectrum, with the proper change of coordinate this gives,

$F_{p, 2 h}\left(\theta_{a}, \varphi_{2}, f_{s}\right)=\frac{4 \pi^{2} f_{s}^{2} \cos \theta_{a} \sin \theta_{a}}{\alpha_{a}^{2}} F_{p, 2 h}\left(\mathbf{K}, f_{s}\right)$

When $\delta_{a}$ terms are kept with eq. (48), the acoustic power radiated by the ocean surface in direction $\varphi_{2}$ can be integrated in any range of incidence angles $\theta_{a, 1}$ to $\theta_{a, 2}$,

$$
\begin{aligned}
P\left(\theta_{a, 1}, \theta_{a, 2}, f_{s}, \varphi_{2}\right)= & \frac{2 \pi^{2} \rho_{a} g^{2}}{\alpha_{a}^{3}} f_{s}^{3} \int_{\theta_{a, 1}}^{\theta_{a, 2}} \sin \theta_{a} \cos \theta_{a} \\
& \times \int_{0}^{2 \pi} \frac{\sigma^{2}\left(\sigma+\sigma^{\prime}\right)\left|R_{a}\right|^{2} E(f, \varphi) E\left(f, \varphi^{\prime}\right)}{\sigma^{\prime 2}\left[\sigma^{\prime}-\sigma \cos \left(\varphi-\varphi^{\prime}\right)\right]} \mathrm{d} \varphi \mathrm{d} \theta_{a},
\end{aligned}
$$

Taking the isotropic form (49), the radiated acoustic power becomes isotropic and the sum over all directions is $2 \pi$ times eq. (51), giving

$2 \pi P\left(\theta_{a, 1}, \theta_{a, 2}, f_{s}, \varphi_{2}\right)=\frac{4 \pi^{3} \rho_{a} g^{2}}{\alpha_{a}^{3}} f_{s}^{3} H\left(f_{s} / 2\right) \int_{\theta_{a, 1}}^{\theta_{a, 2}} \sin \theta_{a} \cos \theta_{a}\left|R_{a}\right|^{2} \mathrm{~d} \theta_{a}$,

with units of $\mathrm{W} \mathrm{m}^{-2} \mathrm{~Hz}^{-1}$, where the so-called 'Hasselmann integral' can be defined from the 'overlap integral' (Farrell \& Munk 2008) and the wave spectrum in frequency,

$H(f)=[E(f)]^{2} I(f)=\int_{0}^{2 \pi} E(f, \varphi) E(f, \varphi+\pi) \mathrm{d} \varphi$. 

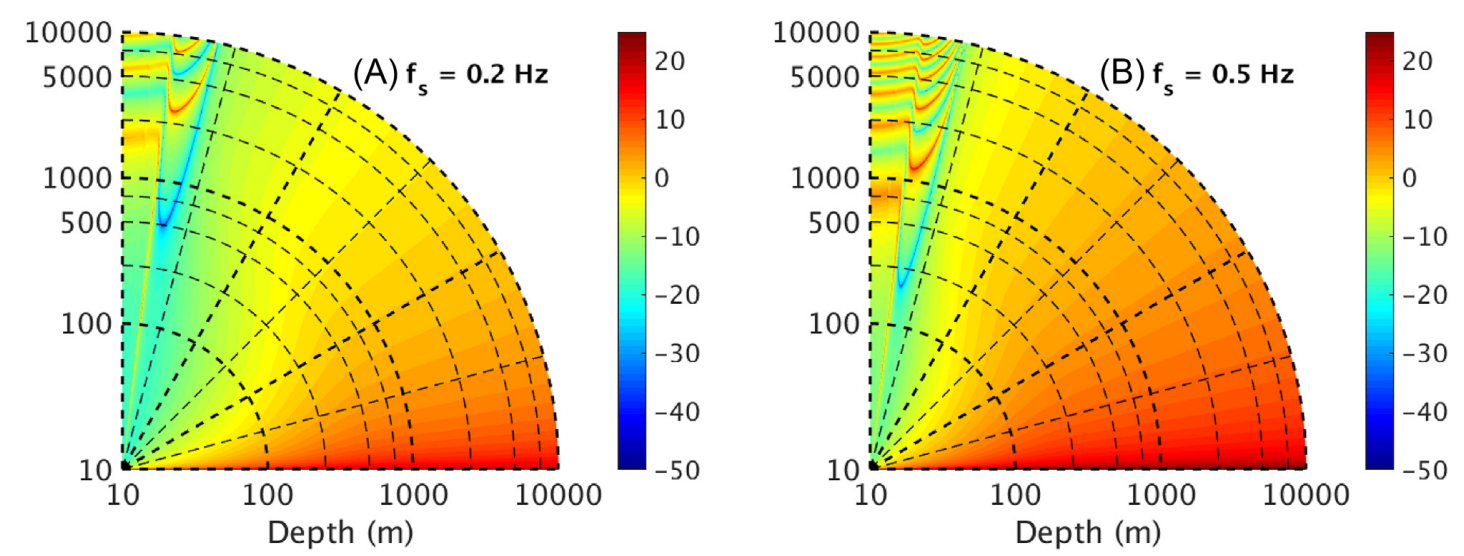

Figure 4. Radiation patterns of $10 \log _{10}\left|R_{a}\right|$ according to eq. (41) for (a) $f_{s}=0.2 \mathrm{~Hz}$ and (b) $f_{s}=0.5 \mathrm{~Hz}$ with $f_{s}=\Omega / 2 \pi$-polar representation against the angle $\theta_{a}$ and the depth $h$.

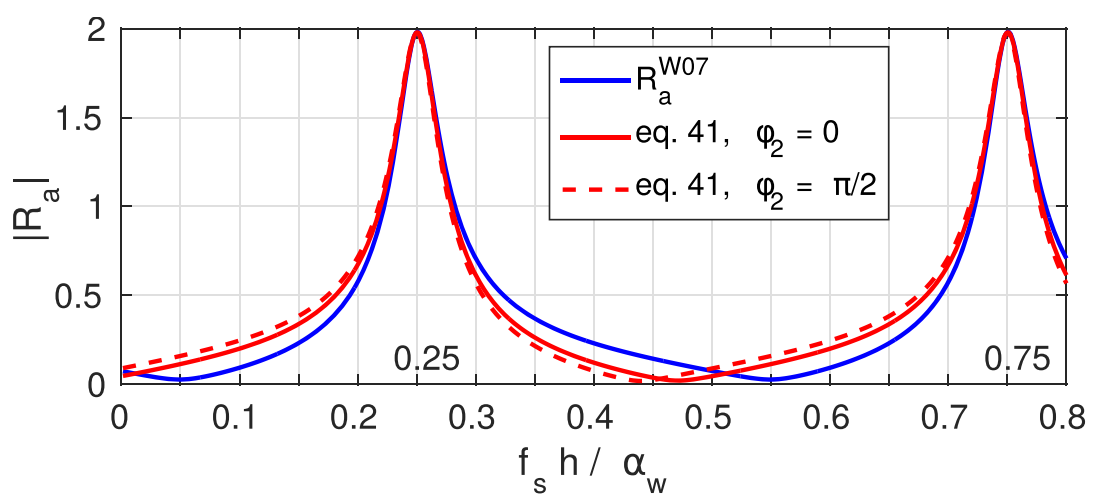

Figure 5. Magnitude of the velocity potential amplification from the water to the air, $R_{a}$, as a function of the ratio of the water depth and acoustic wavelength, in the case of vertical propagation, that is, $\theta_{a}=0$. For $f_{s}=0.2 \mathrm{~Hz}$, the two peaks correspond to depths of 1900 and $5600 \mathrm{~m}$. Here we have used $\alpha_{s}=5540 \mathrm{~m}$ $\mathrm{s}^{-1}, \beta=\alpha_{s} / \sqrt{3}, \rho_{s}=2500 \mathrm{~km} \mathrm{~m}^{-3}$.

The total integrated radiated power, with units of $\mathrm{W} \mathrm{m}^{-2}$, is obtained by integrating eq. (52) across acoustic frequencies $f_{s}$.

\section{PRACTICAL IMPLICATIONS AND DISCUSSION}

In Fig. 6, the mean acoustic intensity over the year 2018 is represented for six ranges of vertical incidence angles. The distribution pattern of sources for the vertical angles $\left[0^{\circ}, 5^{\circ}\right]$ shows the effect of bathymetry, unlike the near-horizontal angles with similar patterns depending almost solely on the Hasselmann integral. For near-vertical angles - $\left[5^{\circ}, 10^{\circ}\right]$ and $\left[10^{\circ}, 15^{\circ}\right]$-the distribution pattern is not continuous; there are resonant points all over the globe. The acoustic intensity is higher for near-horizontal angles, as predicted in Fig. 4.

\subsection{Near-vertical propagation and Rayleigh-wave overestimation}

We note that for vertical propagation $\left(\theta_{a}=0\right)$ the effect of the finite depth changes the amplitude by a factor that ranges from 0.125 to 8.5 with sharp maxima corresponding to the organ pipe resonance at $h=(0.25+n / 2) \alpha_{w} / f_{s}$, as illustrated in Fig. 5, which is similar to fig. 11 in Ardhuin \& Herbers (2013).

The behaviour at other angles is very interesting as $\theta_{a}$ goes through the different regimes of associated seismic waves, from $P$ waves for $\sin \theta_{a}<\alpha_{a} / \alpha_{s}$-that is, $\theta_{a}<3.41^{\circ}$ for our choice of parameters - to Rayleigh waves, which usually contain most of the microseism signal, with $\arcsin \alpha_{a} / \beta<\theta_{a}<\arcsin \alpha_{a} / \alpha_{w}$ corresponding to a range of 5.9 $-12.7^{\circ}$, which is shaded in Fig. 7.

The two maxima that appear in the Rayleigh domain in Fig. 7(a) are the two modes that can exist at $5000 \mathrm{~m}$ depth, whereas only one mode can exist at $1900 \mathrm{~m}$ depth. As discussed by Ardhuin \& Herbers (2013), the depth and frequency at which the amplification is maximum is shifted compared to Fig. 5. This is because the acoustic water component of these Rayleigh modes does not propagate vertically but obliquely at an angle $\theta_{w}$, as shown in Fig. 1(b), giving a resonance at $h=(0.25+n / 2) \alpha_{w} /\left(f_{s} \cos \theta_{w}\right)$.

Our model certainly overestimates the amplitude of these Rayleigh waves and associated microbaroms because we looked for solutions that are homogeneous in space and time. In the solution given above, the leak of energy to the atmosphere is the only loss of energy of the Rayleigh waves and it compensates the source of energy from the local waves. In reality, two important effects limit the microbarom amplitude 

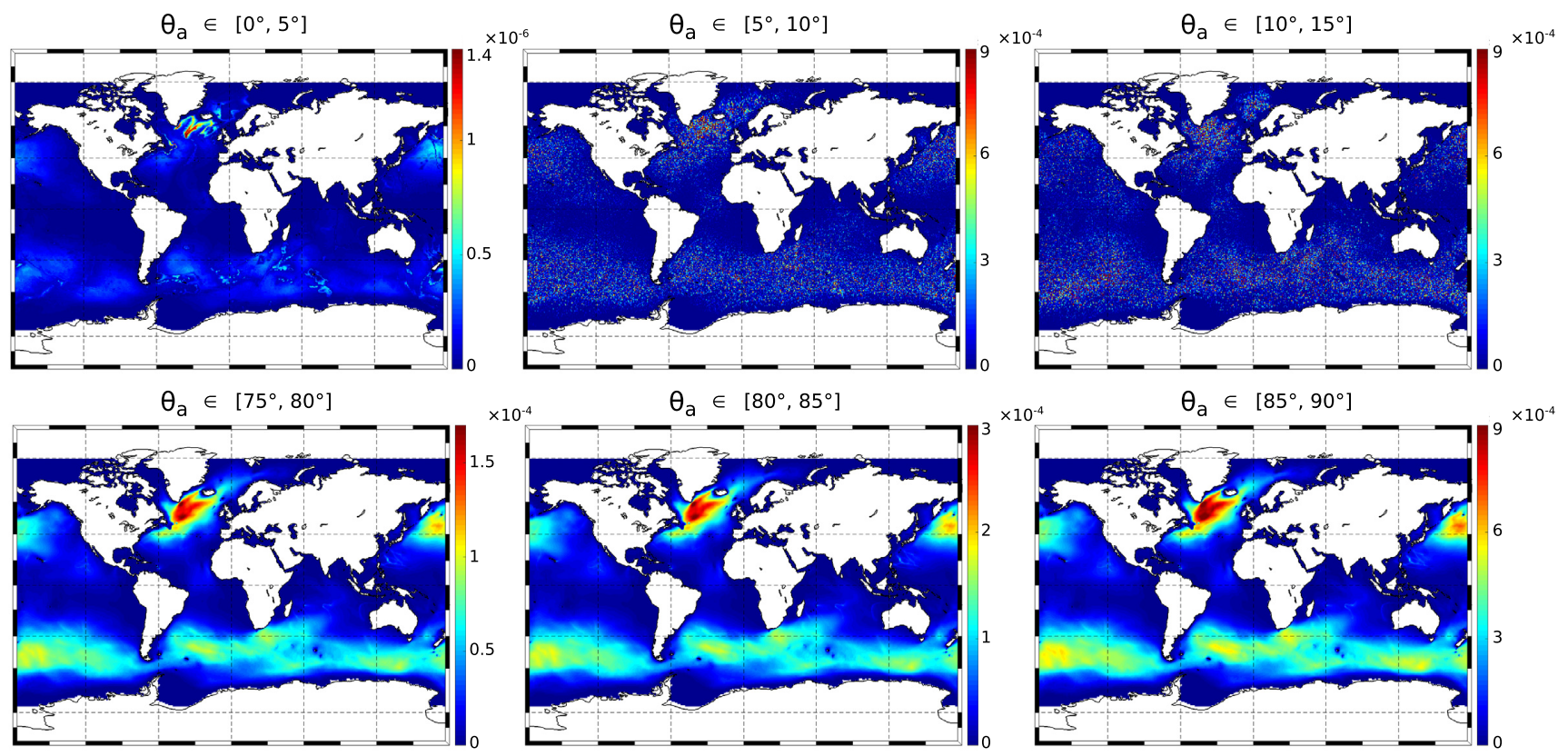

Figure 6. Acoustic intensity in $\mathrm{W} \mathrm{m}^{-2}$ - computed by integrating eq. (52) over $f_{s}$-for six ranges of incidence angles: (a) $\left[\theta_{a, 1}, \theta_{a, 2}\right]=\left[0^{\circ}, 5^{\circ}\right]$, (b) $\left[\theta_{a, 1}\right.$ ,$\left.\theta_{a, 2}\right]=\left[5^{\circ}, 10^{\circ}\right]$, (c) $\left[\theta_{a, 1}, \theta_{a, 2}\right]=\left[10^{\circ}, 15^{\circ}\right]$, (d) $\left[\theta_{a, 1}, \theta_{a, 2}\right]=\left[75^{\circ}, 80^{\circ}\right]$, (e) $\left[\theta_{a, 1}, \theta_{a, 2}\right]=\left[80^{\circ}, 85^{\circ}\right]$ and (f) $\left[\theta_{a, 1}, \theta_{a, 2}\right]=\left[85^{\circ}, 90^{\circ}\right] . \mathrm{Mean}$ of a 3-hourly model over 2018. These were computed from numerical ocean wave model output already described by Rascle \& Ardhuin (2013) and available at $\mathrm{ftp} / / / \mathrm{ftp}$.ifremer.fr/ifremer/ww3/, using the WAVEWATCH III ${ }^{\circledR}$ code (The WAVEWATCH III ${ }^{\circledR}$ Development Group 2016).
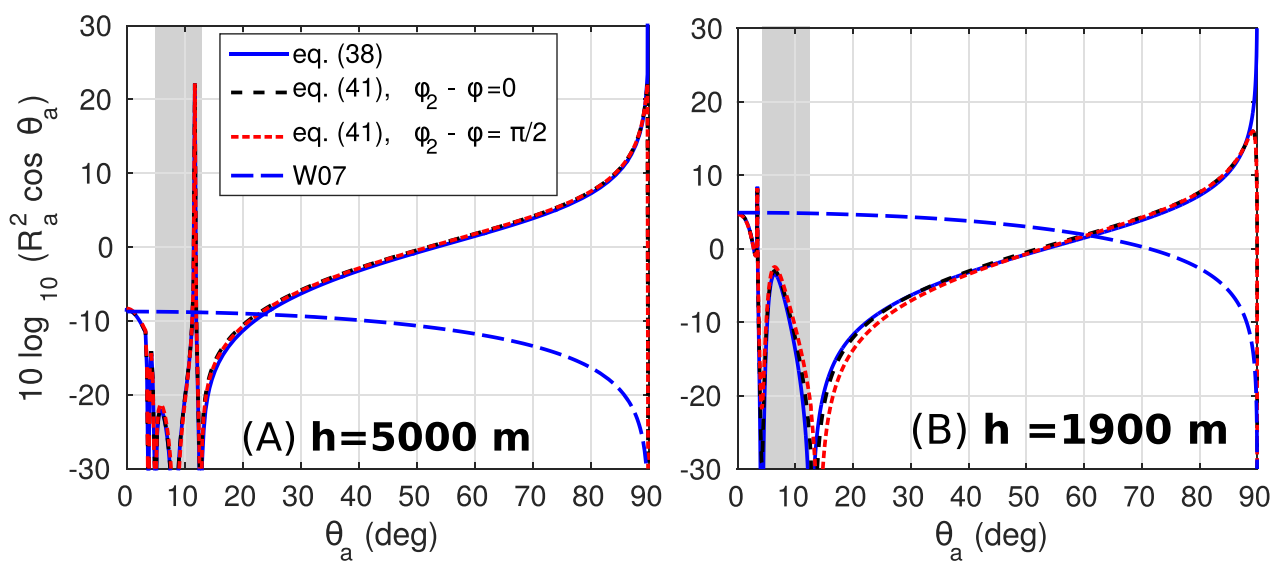

Figure 7. Radiation patterns for an ocean wave period of $10 \mathrm{~s}$, given by the different theories with an ocean bottom at (a) $h=5000 \mathrm{~m}$, (b) $h=1900 \mathrm{~m}$. Note that when the radiated power is considered, these patterns must be multiplied by $\sin \theta_{a}$ before integration over $\theta_{a}$, as given by eq. (52). The shaded region corresponds to the domain of seismic Rayleigh waves with $\arcsin \left(\alpha_{a} / \beta\right)<\theta_{a}<\arcsin \left(\alpha_{a} / \alpha_{w}\right)$.

to a much lower level. First, the ocean storm area of microbarom generation may not be large enough to reach the stationary solution, and second there is a much larger (6-1000 times or more) sink of seismic energy, due to the presence of fluid in the crust, varying with the age of the crust (Stutzmann et al. 2012). The dissipation rate of the energy $E$ is generally parametrized as proportional to $\mathrm{d} E / \mathrm{d} t=-\Omega E / Q_{R}$ where $Q_{R}$ is of the order of 200-1000. Including this effect in this paper amounts to replacing $\Omega$ by $\Omega\left(1-\mathrm{i} /\left(2 Q_{R}\right)\right)$, which is done in Fig. 8 . In other words, the Rayleigh-wave energy is overestimated when dissipation in the crust is neglected because in that case, the leakage of Rayleigh wave energy to the atmosphere is the loss of seismic energy. Hence, our calculation has a seismic attenuation with a very large quality factor $Q_{\max }=\Omega E / S$, where $S$ is the source of seismic energy that equals the radiated power given by eq. (52): $P\left(\arcsin \left(\alpha_{a} / \beta\right), \arcsin \left(\alpha_{a} / \alpha_{w}\right), f_{s}\right)$. Taking twice the kinetic energy in the water column as a lower bound for the total energy we find that, for $h=5000 \mathrm{~m}$ and $f_{s}=0.2 \mathrm{~Hz}, Q_{\max }$ $>10^{6}$ (Supporting Information Section S6.3), meaning that the present solution overestimates the real microbarom amplitude by a factor that exceeds $Q_{\max } / Q_{R}>1000$.

Alternatively, instead of looking for the homogeneous solution to the atmosphere-ocean-crust problem, we can use solutions for the ocean-crust problem with an energy that grows over the source region (Hasselmann 1963; Ardhuin \& Herbers 2013) and compute the microbaroms radiated by microseisms (free Rayleigh waves). These microbaroms are radiated, almost vertically, not only in the source region of microseism but also all along the propagation path of the Rayleigh waves, even on land. For example, a huge microseism with amplitude 

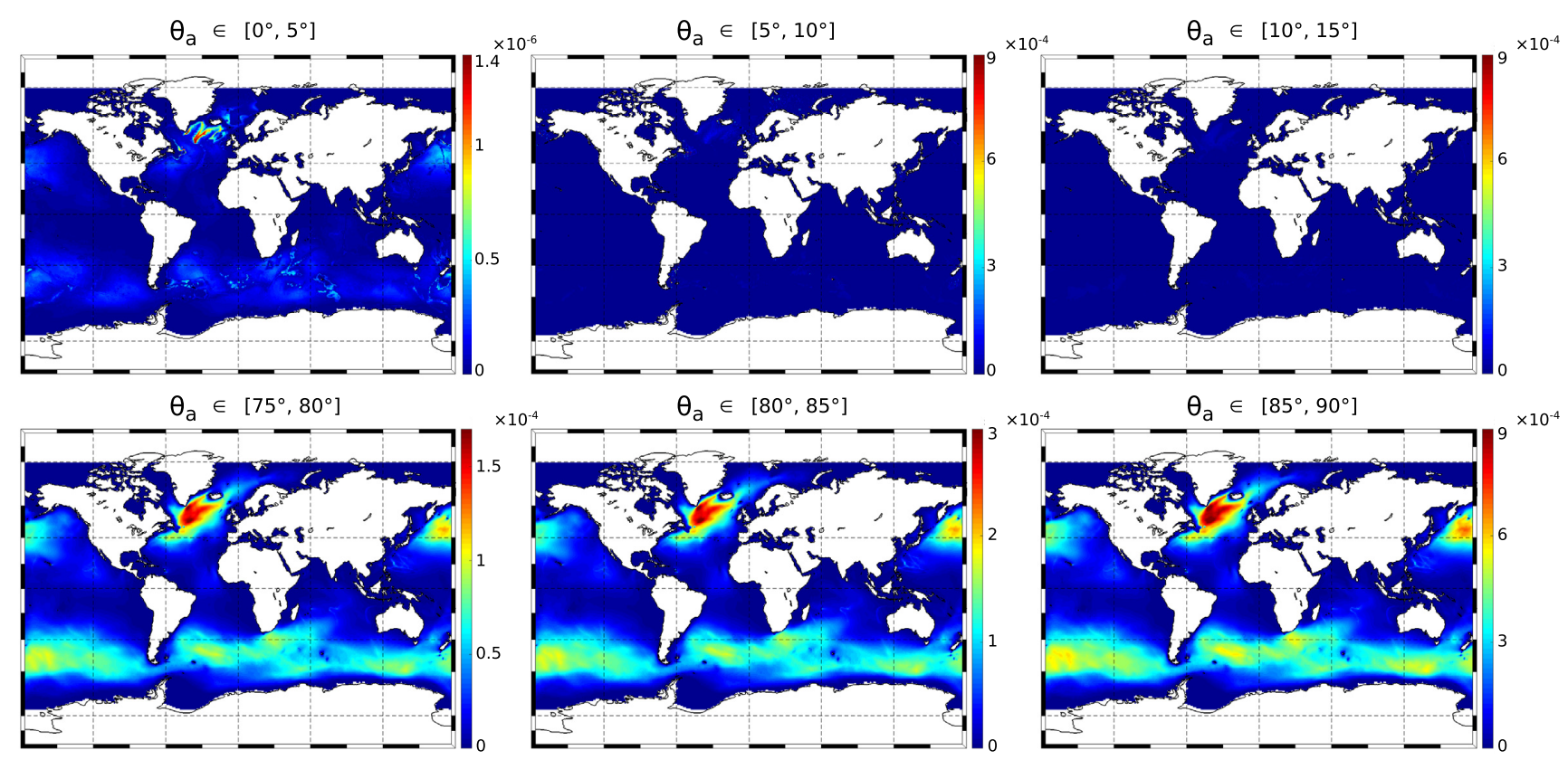

Figure 8. Same as Fig. 6 replacing $\Omega$ by $\Omega\left(1-\mathrm{i} /\left(2 Q_{R}\right)\right.$ with $Q_{R}=201$ in order not to overestimate the Rayleigh-wave energy.

$a=10 \mu \mathrm{m}$ vertical displacement of the sea or land surface corresponds to an energy flux $\rho_{a} \alpha_{a} \Omega^{2} a^{2} / 2$ of only $3 \times 10^{-8} \mathrm{~W} \mathrm{~m}{ }^{-2}$ for $f_{s}=$ $0.2 \mathrm{~Hz}$. This is 2000 times smaller than the peak power measured by Bowman \& Lees (2018). It is thus unlikely that these measurements are dominated by near-vertical propagating sound waves. Indeed, the near-horizontal energy level is usually much stronger.

\subsection{Radiation patterns as a function of azimuth}

The variation of the radiated acoustic power with azimuth $\varphi_{2}$ has not been described before as most studies focused on near-vertical radiation $(K=0)$. In eq. (51), there are two reasons why the radiated power varies with $\varphi_{2}$.

As expressed by the general form given in eq. (51), the radiated power varies with azimuth $\varphi 2$ due to the general form of $R_{a}$, as shown in Figs 3 and 5 but that variation is limited to a few per cent. More important is the fact that the Hasselmann integral $H(f)$ is modified by the interaction of $E(f, \varphi)$ with $E\left(f^{\prime}, \varphi^{\prime}\right)$ instead of $E(f, \varphi+\pi)$, and should be replaced by

$H\left(f, \varphi_{2}\right)=\int_{0}^{2 \pi} \frac{\sigma^{2}\left(\sigma+\sigma^{\prime}\right) E(f, \varphi) E\left(f, \varphi^{\prime}\right)}{\sigma^{\prime 2}\left[\sigma^{\prime}-\sigma \cos \left(\varphi-\varphi^{\prime}\right)\right]} \mathrm{d} \varphi$.

Following the classification in Ardhuin et al. (2011), the oceanic conditions in which the Hasselmann integral takes the largest values correspond to 'class III' events, with two narrow swells propagating in opposite directions. These are also conditions in which $E\left(f^{\prime}, \varphi^{\prime}\right)$ may be most different from $E(f, \varphi+\pi)$.

\subsubsection{Case of analytical ocean wave spectra}

To illustrate this effect, we take an example of a family of wave spectra adapted from the case discussed in Obrebski et al. (2012). This family of spectra is defined by the analytic expression

$E(f, \varphi)=E_{0}\left[\mathrm{e}^{-\frac{\left(f-f_{1}\right)^{2}}{2 f_{2}^{2}}-\frac{\left(\varphi-\varphi_{1}\right)^{2}}{2 \varphi_{3}^{2}}}+\mathrm{e}^{-\frac{\left(f-f_{3}\right)^{2}}{2 f_{4}^{2}}-\frac{\left(\varphi-\varphi_{4}\right)^{2}}{2 \varphi_{5}^{2}}}\right]$,

where the $f_{1}, f_{2}, f_{3}$, and $f_{4}$ parameters define the peak frequency and width for the two swell trains, and $\varphi_{1}, \varphi_{3}, \varphi_{4}, \varphi_{5}$ define the mean direction and width. The present theory is not restricted to this family of spectra and generally applies to any wave spectrum. Such an analytical form is particularly useful for testing the influence of the discretization when the spectrum is given by a numerical model.

Fig. 9(a) shows this spectrum transformed to wavenumber space $E\left(k_{x}, k_{y}\right)=g^{2} E(f, \varphi) / 4 \pi \sigma^{3}$, with the mean frequency and direction set to $f_{1}=0.066 \mathrm{~Hz}, f_{3}=0.066 \mathrm{~Hz}, \varphi_{1}=90^{\circ}, \varphi_{3}=270^{\circ}$, the frequency and direction widths set to $f_{2}=0.007 \mathrm{~Hz}, f_{4}=0.007 \mathrm{~Hz}, \varphi_{4}=8^{\circ}, \varphi_{5}$ $=8^{\circ}$, and the normalization factor $E_{0}=20 \mathrm{~m}^{2} / \mathrm{Hz}$, giving a significant wave height of $2 \mathrm{~m}$.

The azimuthal variation of the generalized Hasselmann integral, as given by eq. (54), is illustrated in Fig. 9(b). 

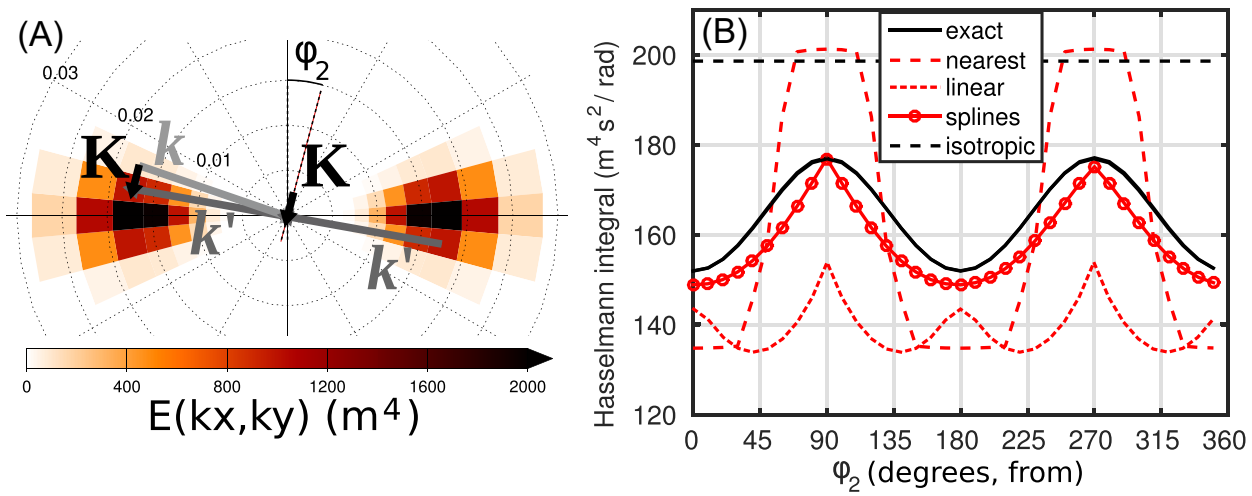

Figure 9. (a) Example spectrum discretized with 36 directions and frequency exponentially spaced with an 1.1 increment from one frequency to the next, typically used in numerical wave models. The spectral density of the ocean waves is shown in colours, in directions from where the waves come. (b) Resulting integrals for the acoustic frequency $f_{s}=0.13 \mathrm{~Hz}$, and horizontal propagation $\left(\theta_{a}=90^{\circ}, K=0.0025 \mathrm{rad} \mathrm{m}^{-1}\right)$. Three methods were used to compute the integral: the analytic spectral expression or interpolation of the discrete spectrum using nearest neighbour or linear interpolation.

The exact calculation uses the analytic expression of the spectrum and exhibits variations of 7 per cent of the radiated acoustic power as a function of $\varphi_{2}$, with a maximum in the direction of the waves because the spectrum in $k$-space is more narrow in the $k_{y}$ than in the $k_{x}$ direction and when $K$ is aligned with the $y$-axis; as shown, the wavenumber vector $\mathbf{k}^{\prime}$ falls away from the peak faster than when $K$ is aligned with the $x$-axis. For the present example the azimuthal variation goes away when the directional spread is increased from $8^{\circ}$ to $12^{\circ}$ and it has a maximum at $0^{\circ}$ and $180^{\circ}$ for wider directional spectra.

The practical estimation of the integral is very sensitive to the discretization used, which is not an issue when $K$ is much smaller than the discretization of the spectrum and the isotropic form can be used. Numerical wave models that typically use 10 per cent increments from one frequency to the next and 24 or 36 directions do not resolve very well the narrow swell peaks such as those in Fig. 9(a). As a result, a linear interpolation underestimates the integral because the peak appears narrower. On average this is corrected by using the nearest point, but that approach can exaggerate the anisotropy of the acoustic source.

Besides causing anisotropic sources when two swell peaks are exactly opposed, the generalized integral may broaden the region where sources are significant, as shown in Fig. 10, because it allows a wider range of directions and frequencies to interact compared to $\mathbf{k}=\mathbf{k}^{\prime}$ in the simplified form.

\subsubsection{General case using numerical wave model output}

In order to test this idea, we have computed the Hasselmann integral from wave-model output which is 'real' spectrum (non-idealized spectrum) with the usual form and its generalization in the case of the event discussed by Bowman \& Lees (2018). Fig. 11 shows modelled maps of wave source magnitude, in colour, using the isotropic or azimuth-dependent form of the Hasselmann integral over a $10^{\circ} \times 10^{\circ}$ region located to the southeast of New Zealand, valid for May 2016, at 06:00 UTC. The wave model used here is very similar to the one used in Ardhuin et al. (2015), with a number of discrete directions increased from 24 to 36. This strong microbarom source is associated with a strong local wind, up to $18 \mathrm{~m} \mathrm{~s}^{-1}$, blowing against swell coming from a remote storm, typical of a class-III event described by Ardhuin $e t$ al. (2011).

The first striking result is that the colours are very similar, with a correlation of 0.9998 , meaning that the simplified isotropic form is a good approximation of the total radiated power, at least for this example. We also note that the highest sources are most isotropic, such as at location D in Fig. 11(b). Where sources are more strongly radiated in one direction, such as at locations $\mathrm{C}$ and $\mathrm{E}$, this is due to a gradual shift in the direction of both interacting wave trains, the swell from the southwest has a local direction that is close to $240^{\circ}$ at $\mathrm{C}$, and $255^{\circ}$ at $\mathrm{E}$, and the time evolution of the local wind means that the wind sea is rather from the east at $\mathrm{C}$ and the northeast at $\mathrm{E}$. In this particular example, this gives a dominant radiation from the southeast at $\mathrm{C}$ and the north at $\mathrm{E}$.

When averaged over the entire area, the difference between the radiated power in any given direction and the isotropic solution is less than 15 per cent, suggesting that the isotropic approximation may be accurate enough for most applications.

\section{CONCLUSIONS}

In this paper we have reviewed and unified the microbarom source theories developed by Brekhovskikh et al. (1973) and Waxler et al. (2007). A prominent feature of Brekhovskikh et al. (1973), that was not taken into account by Waxler et al. (2007), is the radiation pattern as a function of the elevation angle $\theta_{a}$. In Waxler et al. (2007), the radiation pattern is monopolar due to an assumed lack of coherence of the sources at scales comparable to the acoustic wavelength. In Brekhovskikh et al. (1973), the acoustic power at near-horizontal incidence is up to 1000 times larger because the main coupling of water and air via the vertical velocity of the air-sea interface gives a much stronger amplification for grazing angles. Including finite depth ocean effects in Brekhovskikh et al. (1973) model is relevant for near-vertical 

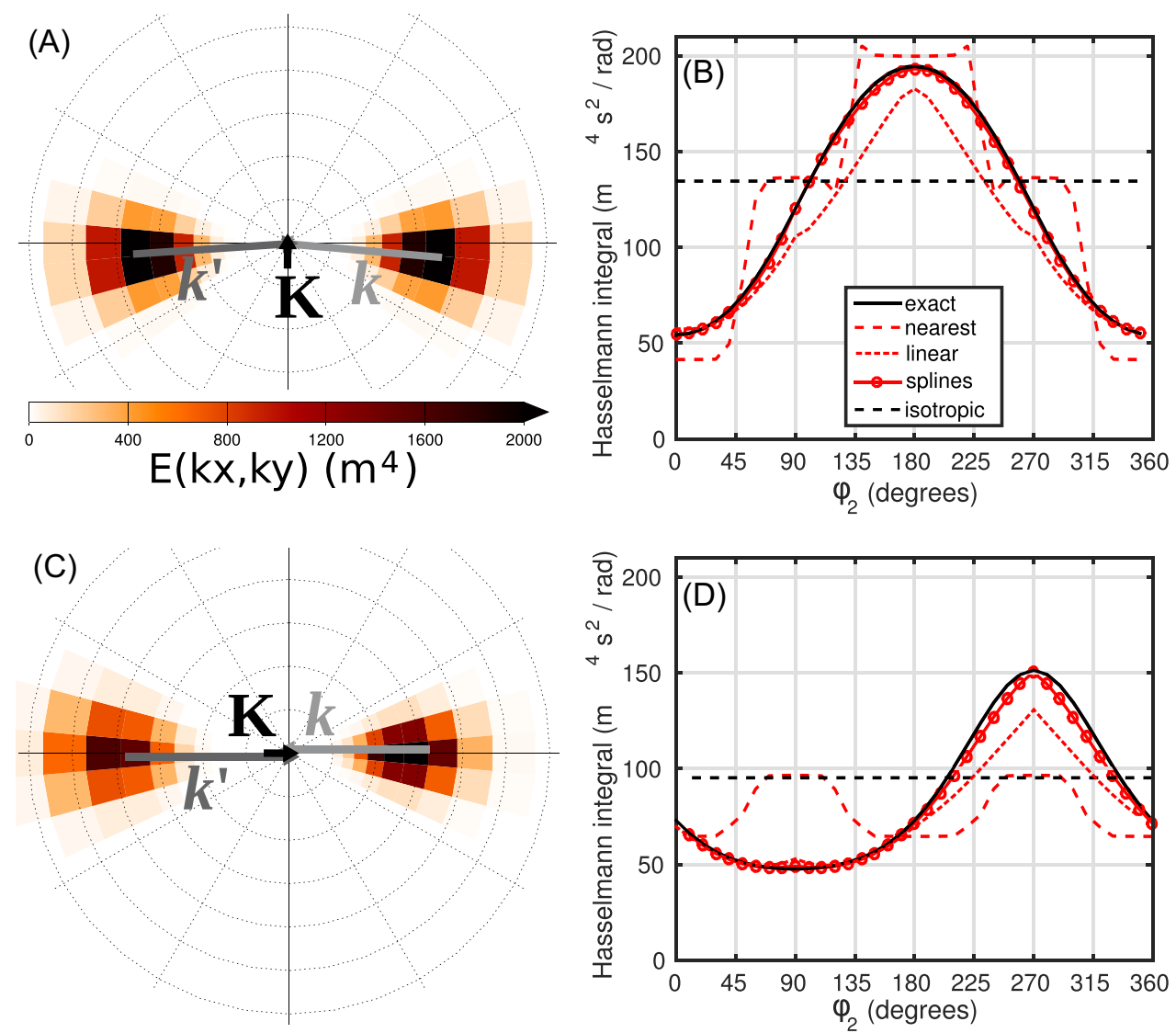

Figure 10. Same as Fig. 9 with different ocean wave spectra (a) that have mean directions shifted by $5^{\circ}$ to $\varphi_{1}=95^{\circ}$ and $\varphi_{3}=265^{\circ}$ producing the Hasselmann integral in (b). The wave spectrum produces the Hasselmann integral in (d), with peak frequencies shifted by $0.006 \mathrm{~Hz}$ to $f_{1}=0.072 \mathrm{~Hz}$ and $f_{2}=0.060 \mathrm{~Hz}$. For both cases, the configuration of $\mathbf{k}$ and $\mathbf{k}^{\prime}$ gives the largest contribution to the Hasselmann integral indicated in (a) and (c) with arrows.

propagation only, whereas for near-horizontal propagation it has almost no effect. For shallow propagation angles that generally correspond to ground-based measurements (i.e. $\theta_{a}>40^{\circ}$, relative to vertical), Brekhovskikh et al. (1973)'s formulation is compared to the more complete depth-dependent model presented here. We find that in regions of water depths under $1000 \mathrm{~m}$, which cover 10 per cent of the total ocean surface, Brekhovskikh et al. (1973)'s formulation overestimates the source amplitude and underestimates it for deeper waters. On average there is a 7 per cent underestimation and Brekhovskikh et al. (1973) give overall acceptable results, with a negligible effect of the water depth for near-horizontal propagation angles.

For near-vertical propagation, the generation of microbaroms involves both a source mechanism similar to the one of microseism dominated by the nonlinear interaction of near-opposing waves, and Rayleigh waves propagating away from microseism sources. However, the associated acoustic power is at least 2 orders of magnitude lower than the near-horizontal radiated power. These vertical angles can be of interest for altitude measurements such as balloon measurements (Bowman \& Lees 2018); in such a situation, the bathymetry can still impact the received signal. The horizontal anisotropy of the source has also been investigated, leading to the conclusion that, for computational applications, the isotropic approximation may be accurate enough and could then be used. Hence, the discretization of the wave spectrum might not be an issue.

Beyond theoretical issues, efforts should be pursued to validate the proposed model by considering available observations of infrasound ambient noise as recorded by the global infrasound network of the International Monitoring System (IMS; Ceranna et al. 2019). For practical applications, further developments of a numerical model are needed to propagate microbarom signals over large distances through a realistic atmosphere. The implementation of this source model, based on a state-of-the-art numerical wave model (Ardhuin 2019), should help building a global and time-dependent reference database. Exploiting this database of oceanic noise sources will be useful for developing middle-atmospheric remote sensing methods. The evaluation of infrasound ambient noise model is essential in the context of the future verification of the Comprehensive nuclear Test Ban Treaty, as accurate atmospheric models are basic prerequisite to assess the IMS network performance in higher resolution, reduce source location errors and improve source characterization methods. 


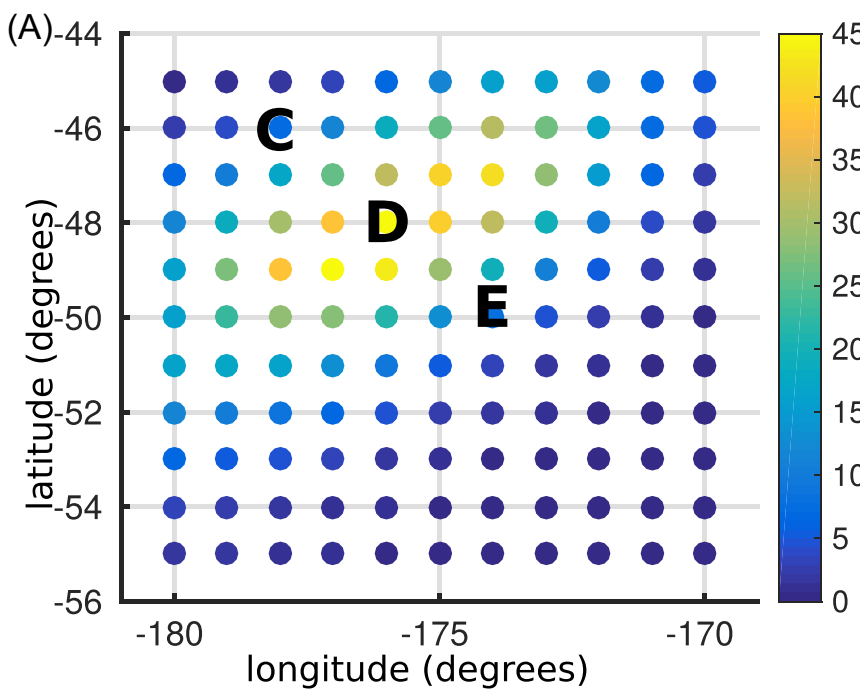

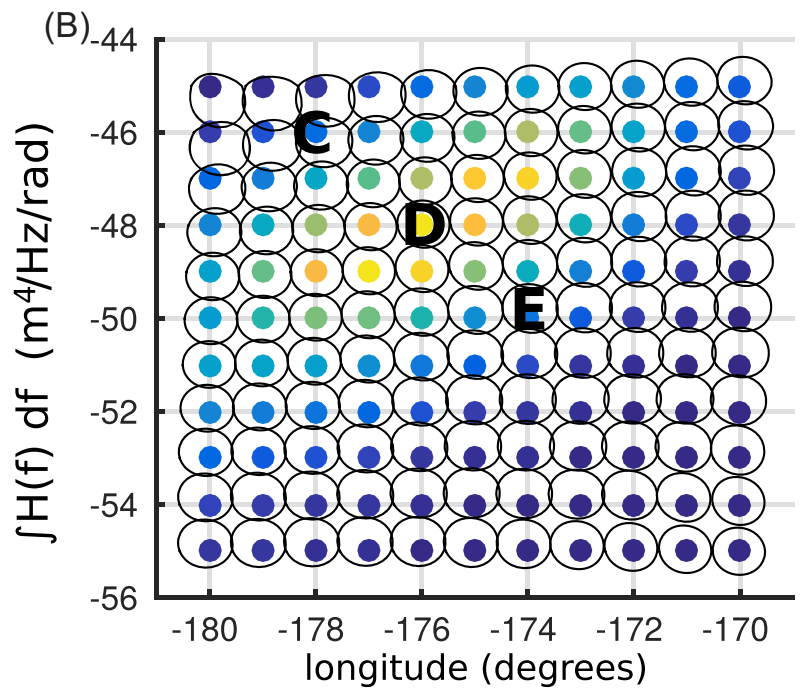

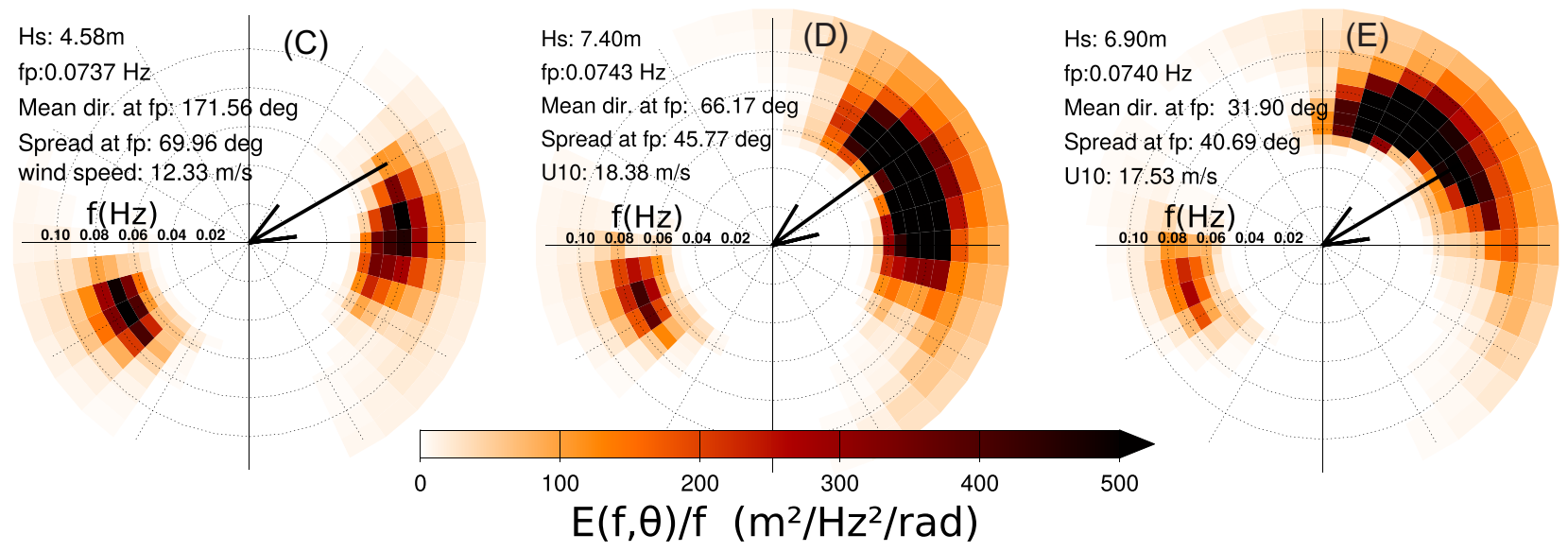

Figure 11. Maps of the Hasselmann Integral for (a) the isotropic and (b) non-isotropic expressions. In (b), the black curve around each location represents the Hasselmann integral estimated as a function of the acoustic propagation azimuth, and plotted in direction from where the acoustic waves are coming and normalized so that the average radius is half the distance between neighbouring locations. (c), (d) and (e) are showing modelled directional spectra at the C, D, and $\mathrm{E}$ locations mentioned in (a) and (b). The large arrows indicate the wind direction, from the northeast, associated with a broad wind sea spectrum which opposes a more narrow swell spectrum from the southwest.

\section{ACKNOWLEDGEMENTS}

The ocean wave spectra from which the Hasselmann integral were computed with the WAVEWATCH III wave-action model, and these spectra are available at ftp://ftp.ifremer.fr/ifremer/ww3/.

The research leading to these results was partly performed during the course of the ARISE2 project (http://ARISE-project.eu) funded by the European Commission Horizon 2020 (grant agreement 65398). This work was also supported by Agence Nationale de la Recherche under grant ANR-14-CE01-0012 'MIMOSA'.

This paper owes much to discussion with Roger Waxler, his review and the review of one anonymous reviewer.

\section{REFERENCES}

Ardhuin, F., 2019. Ocean waves in geosciences, doi:10.13140/RG.2.2.16019.78888/5.

Ardhuin, F. \& Herbers, T.H.C., 2013. Noise generation in the solid earth, oceans and atmosphere, from nonlinear interacting surface gravity waves in finite depth, J. Fluid Mech., 716, 316-348.

Ardhuin, F., Stutzmann, E., Schimmel, M. \& Mangeney, A., 2011. Ocean wave sources of seismic noise, J. geophys. Res., 116, C09004, doi:10.1029/2011JC006952.

Ardhuin, F. et al., 2013. A numerical model for ocean ultra low frequency noise: wave-generated acoustic-gravity and Rayleigh modes, J. acoust. Soc. Am., 134(4), 3242-3259.
Ardhuin, F., Gualtieri, L. \& Stutzmann, E., 2015. How ocean waves rock the earth: two mechanisms explain seismic noise with periods 3 to $300 \mathrm{~s}$, Geophys. Res. Lett., 42, 765-772.

Ardhuin, F., , Gualtieri, L. \& Stutzmann, E., 2019. Physics of ambient noise generation by ocean waves, in Surface Waves and Fluxes, pp. 69-108, eds Nakata, N., Gualtieri, L. \& Fichtner, A., Cambridge University Press.

Assink, J., Pichon, A.L., Blanc, E., Kallel, M. \& Khemiri, L., 2014. Evaluation of wind and temperature profiles from ECMWF analysis on two hemispheres using volcanic infrasound, J. geophys. Res., 119(14), 86598683.

Benioff, H. \& Gutemberg, B., 1939. Waves and currents recorded by electromagnetic barographs, Bull. seism. Soc. Am., 20(10), 421-426. 
Blanc, E. et al., 2018. Toward an improved representation of middle atmospheric dynamics thanks to the arise project, Surv. Geophys., 39(2), $171-225$.

Bowman, D.C. \& Lees, J.M., 2018. Upper atmosphere heating from oceangenerated acoustic wave energy, Geophys. Res. Lett., 45, 5144-5150.

Brekhovskikh, L.M., Goncharov, V.V., Kurtepov, V.M. \& Naugolnykh, K.A., 1973. The radiation of infrasound into the atmosphere by surface waves in the ocean, Izv. Atmos. Ocean. Phys., 9, 899-907( In the English translation, 511-515).

Bromirski, P.D., Flick, R.E. \& Graham, N., 1999. Ocean wave height determined from inland seismometer data: implications for investigating wave climate changes in the NE Pacific, J. geophys. Res., 104(C9), 20 753-20 766.

Ceranna, L., Matoza, R., Hupe, P., Le Pichon, A. \& Landès, M., 2019. Systematic array processing of a decade of global IMS infrasound data, in Infrasound Monitoring for Atmospheric Studies, pp. 471-482, eds Le Pichon, A., Blanc , E. \& Hauchecorne, A., Springer.

Cox, C.S. \& Jacobs, D.C., 1989. Cartesian diver observations of double frequency pressure fluctuations in the upper levels of the ocean, Geophys. Res. Lett., 16(8), 807-810.

Donn, W.L. \& Naini, B., 1973. Sea wave origin of microbaroms and microseism, J. geophys. Res., 78(21), 4482-4488.

Donn, W.L. \& Rind, D., 1971. Natural infrasound as an atmospheric probe, Geophys. J. R. astr. Soc., 26(1), 111-133.

Drob, D., 2019. Meteorology, climatology, and upper atmospheric composition for infrasound propagation modeling, in Infrasound Monitoring for Atmospheric Studies, pp. 485-508, eds Le Pichon, A., Blanc, E. \& Hauchecorne, A., Springer.

Drob, D.P., Meier, R., Picone, J.M. \& Garcés, M.M., 2010. Inversion of infrasound signals for passive atmospheric remote sensing, in Infrasound Monitoring for Atmospheric Studies, pp. 701-731, eds Le Pichon, A., Blanc, E. \& Hauchecorne, A., Springer.

Farrell, W.E. \& Munk, W., 2008. What do deep sea pressure fluctuations tell about short surface waves?, Geophys. Res. Lett., 35(7), L19605, doi:10.1029/2008GL035008.

Garcés, M., Drob, D.P. \& Picone, J.M., 2002. A theoretical study of the effect of geomagnetic fluctuations and solar tides on the propagation of infrasonic waves in the upper atmosphere, Geophys. J. Int., 148, 77-87.

Gualtieri, L., Stutzmann, E., Farra, V., Capdeville, Y., Schimmel, M., Ardhuin, F. \& Morelli, A., 2014. Modelling the ocean site effect on seismic noise body waves, Geophys. J. Int., 193, 1096-1106.

Hasselmann, K., 1962. On the non-linear energy transfer in a gravity wave spectrum, part 1: general theory, J. Fluid Mech., 12, 481-501.

Hasselmann, K., 1963. A statistical analysis of the generation of microseisms, Rev. Geophys., 1(2), 177-210.
Hasselmann, K., 1966. Feynman diagrams and interaction rules of wave-wave scattering processes, Rev. Geophys., 4(1), 1-32, doi:10.1029/2009JB006918.

Landès, M., Hubans, F., Shapiro, N.M., Paul, A. \& Campillo, M., 2010. Origin of deep ocean microseisms by using teleseismic body waves, $J$. geophys. Res., 115, $\mathrm{B} 05302$.

Le Pichon, A. et al., 2015. Comparison of co-located independent groundbased middle atmospheric wind and temperature measurements with numerical weather prediction models, J. geophys. Res., 120(16), 8318-8331.

Longuet-Higgins, M.S., 1950. A theory of the origin of microseisms, Phil. Trans. R. Soc. Lond., A, 243, 1-35.

Marty, J., 2019. The IMS infrasound network: current status and technological developments, in Infrasound Monitoring for Atmospheric Studies, pp. 3-62, eds Le Pichon, A., Blanc , E. \& Hauchecorne, A., Springer.

Meschede, M., Stutzmann, E., Farra, V., Schimmel, M. \& Ardhuin, F., 2017. The effect of water-column resonance on the spectra of secondary microseism P-waves, J. geophys. Res., 122, 8121-8142.

Obrebski, M., Ardhuin, F., Stutzmann, E. \& Schimmel, M., 2012. How moderate sea states can generate loud seismic noise in the deep ocean, Geophys. Res. Lett., 39, L11601, doi:10.1029/2012GL051896.

Obrebski, M., Ardhuin, F., Stutzmann, E. \& Schimmel, M., 2013. Detection of microseismic compressional $(P)$ body waves aided by numerical modeling of oceanic noise sources, J. geophys. Res., 118, 4312-4324.

Posmentier, E., 1967. A theory of microbaroms, Geophys. J. R. astr. Soc., 13, 487-501.

Rascle, N. \& Ardhuin, F., 2013. A global wave parameter database for geophysical applications. Part 2: Model validation with improved source term parameterization, Ocean Modelling, 70, 174-188.

Smets, P.S.M. \& Evers, L.G., 2014. The life cycle of a sudden stratospheric warming from infrasonic ambient noise observations, J. geophys. Res., 119, 12 084-12 099.

Stutzmann, E., Schimmel, M. \& Ardhuin, F., 2012. Modeling long-term seismic noise in various environments, Geophys. J. Int., 191, 707-722.

The WAVEWATCH III ${ }^{\circledR}$ Development Group, 2016. User manual and system documentation of WAVEWATCH III ${ }^{\circledR}$ version 5.16 , Tech. Note 329, NOAA/NWS/NCEP/MMAB, College Park, MD, USA, 326 pp. + Appendices.

Waxler, R. \& Assink, J., 2019. Propagation modeling through realistic atmosphere and benchmarking, in Infrasound Monitoring for Atmospheric Studies, pp. 509-549, eds Le Pichon, A., Blanc , E. \& Hauchecorne, A., Springer.

Waxler, R. \& Gilbert, K.E., 2006. The radiation of atmospheric microbaroms by ocean waves, J. acoust. Soc. Am., 119, 2651-2664.

Waxler, R., Gilbert, K., Talmadge, C. \& Hetzer, C., 2007. The effects of finite depth of the ocean on microbarom signals, in 8th Int. Conf. Theoretical and Computational Acoustics (ICTCA), Crete, Greece.

\section{SUPPORTING INFORMATION}

Supplementary data are available at $G J I$ online.

\section{SI_GJI_revised_DeCarlo_et_al19_cleanfile.pdf}

Table S1. Notations used in different papers: LH50 stands for Longuet-Higgins (1950), BGKN73 stands for Brekhovskikh et al. (1973), WG06 stands for Waxler \& Gilbert (2006) and AH13 stands for Ardhuin \& Herbers (2013).

Please note: Oxford University Press is not responsible for the content or functionality of any supporting materials supplied by the authors. Any queries (other than missing material) should be directed to the corresponding author for the paper.

\section{APPENDIX: GREEN FUNCTION PHASE, SPATIAL CORRELATIONS AND TREATMENT BY WAXLER \& GILBERT (2006)}

In order to clarify the difference between our derivation and the derivation by Waxler \& Gilbert (2006), we go back to their expression of the atmospheric pressure spectrum as recorded at the horizontal position $\mathbf{x}_{\mathrm{H}}$ and vertical altitude $z$, from a collection of sources over positions $y$ 
and $y^{\prime}$ in domain $S$. The variance of pressure is given by their eq. (49) with $\mathbf{x}_{\mathrm{H}}^{\prime}=\mathbf{x}_{\mathrm{H}}, z^{\prime}=z$ and $\tau=0$

$\left\langle P^{2}\right\rangle=\rho^{2} \int_{-\infty}^{\infty} \int_{-\infty}^{\infty} \int_{S} \int_{S}\left\langle G\left(x, y,-\tau_{1}\right) G^{*}\left(x, y^{\prime}, \tau-\tau_{2}\right)\right\rangle\left\langle\frac{\partial v\left(y, \tau_{1}\right)}{\partial \tau_{1}} \frac{\partial v\left(y^{\prime}, \tau_{2}\right)}{\partial \tau_{2}}\right\rangle_{S} \mathrm{~d}^{2} y \mathrm{~d}^{2} y^{\prime} \mathrm{d} \tau_{1} \mathrm{~d} \tau_{2}$.

Following their derivation, we use the Fourier in time $\widehat{G}$ of Green's function, representing the propagation of acoustic waves in a layered medium as a sum of discrete modes. As given by Waxler \& Gilbert (2006; eq. 65) the Fourier transform of the Green function for mode $j$ and frequency $v$ is given by

$\widehat{G}\left(\mathbf{x}_{\mathrm{H}}, z, \mathbf{y}_{\mathrm{H}}, v\right)=f(r, z) \sum_{j} \frac{\mathrm{e}^{\mathrm{i} k_{j}\left|\mathbf{x}_{\mathrm{H}}-\mathbf{y}_{\mathrm{H}}\right|}}{\sqrt{k_{j}}} \psi_{j}(z) \psi_{j}\left(z^{\prime}\right)$,

where $r=\left|\mathbf{x}_{\mathrm{H}}-\mathbf{y}_{\mathrm{H}}\right|$ is the horizontal distance, the $k_{j}$ are the horizontal wavenumbers pointing in the direction of $\widehat{\mathbf{k}}=\left(\mathbf{x}_{\mathrm{H}}-\mathbf{y}_{\mathrm{H}}\right) / r$, and $\psi_{j}$ are normal modes satisfying the bi-orthogonality condition and their phases are assumed to be uniformly distributed and statistically independent.

Considering that for each $y$ in the source $\left|\mathbf{y}_{\mathrm{H}}^{\prime}-\mathbf{y}_{0}\right| /\left|\mathbf{x}_{\mathrm{H}}-\mathbf{y}_{0}\right| \ll 1$ with $y_{0}$ the centre of the source (i.e. the receptor is far from the source), and defining the vector $\mathbf{k}_{j}=k_{j} \widehat{\mathbf{k}}$, the product of Green's function for $\mathbf{y}_{\mathrm{H}}$ and its complex conjugate for $\mathbf{y}_{\mathrm{H}}^{\prime}$ writes

$\widehat{G}\left(\mathbf{x}_{\mathrm{H}}, z, \mathbf{y}_{\mathrm{H}}, v\right) \widehat{G}\left(\mathbf{x}_{\mathrm{H}}, z, \mathbf{y}_{\mathrm{H}}^{\prime}, v\right)^{\star}=\left|f\left(r_{0}, z\right)\right|^{2} \sum_{j} \mathrm{e}^{\left.\mathrm{i} \mathbf{k}_{j} \cdot \mathbf{y}^{\prime}-\mathbf{y}\right)} \frac{\left|\psi_{j}(z)\right|^{2}\left|\psi_{j}\left(z^{\prime}\right)\right|^{2}}{k_{j}}$.

The phase of this product then contains the phase shift of the propagation from $\mathbf{y}$ to $\mathbf{y}^{\prime}$ with a wavenumber $k_{j}$. When correlated with the source structure, that contains a phase $\exp \left[i(\mathbf{k}+\mathbf{q}) \cdot\left(\mathbf{y}^{\prime}-\mathbf{y}\right)\right]$ it gives,

$$
\begin{aligned}
\left\langle P^{2}\right\rangle= & \rho^{2} \int_{S} \int_{S}\left(\left|f\left(r_{0}, z\right)\right|^{2} \sum_{j} \frac{\mathrm{e}^{i \mathbf{k}_{\mathbf{j}}\left(\mathbf{y}^{\prime}-\mathbf{y}\right)}}{\left|k_{j}\right|}\left|\psi_{j}(z)\right|^{2}\left|\psi_{j}\left(z^{\prime}\right)\right|^{2}\right) \\
& \times\left(2 \iint v^{2} F(\mathbf{k}) F(\mathbf{q})\left|C^{+}\right|^{2}|\Omega|^{2} \mathrm{e}^{i(\mathbf{k}+\mathbf{q})\left(y-y^{\prime}\right)} \mathrm{d}^{2} \mathbf{k} \mathrm{d}^{2} \mathbf{q}\right) \mathrm{d}^{2} y \mathrm{~d}^{2} y^{\prime} \\
= & \rho^{2} \int_{S} \int_{S}\left|f\left(r_{0}, z\right)\right|^{2} \sum_{j} \iint\left[\frac{\left|\psi_{j}(z)\right|^{2}\left|\psi_{j}\left(z^{\prime}\right)\right|^{2}}{\left|k_{j}\right|} \mathrm{e}^{i\left(\mathbf{k}_{\mathbf{j}}-(\mathbf{k}+\mathbf{q})\right)\left(\mathbf{y}^{\prime}-\mathbf{y}\right)}\right. \\
& \left.\times 2 v^{2} F(\mathbf{k}) F(\mathbf{q})\left|C^{+}\right|^{2}|\Omega|^{2}\right] \mathrm{d}^{2} \mathbf{k} \mathrm{d}^{2} \mathbf{q} \mathrm{d}^{2} y \mathrm{~d}^{2} y^{\prime} .
\end{aligned}
$$

This can be simplified as the integral over $\mathbf{y}^{\prime}$ in the source area $S$ gives a term proportional to

$$
\int_{S} \int_{S} \mathrm{e}^{\mathrm{i}\left[\left(\mathbf{k}_{j}-(\mathbf{k}+\mathbf{q})\right) \cdot\left(\mathbf{y}_{H}-\mathbf{y}_{H}^{\prime}\right)\right]} \mathrm{d}^{2} y \mathrm{~d}^{2} y^{\prime}=(2 \pi)^{2} \delta\left(\mathbf{k}_{\mathbf{j}}-(\mathbf{k}+\mathbf{q})\right) S
$$

instead of the $(2 \pi)^{2} \delta(\mathbf{k}+\mathbf{q})$ term found by Waxler \& Gilbert (2006).

The pressure variance becomes

$$
\left\langle P^{2}\right\rangle=\rho^{2}(2 \pi)^{2} S\left|f\left(r_{0}, z\right)\right|^{2} \sum_{j} \int_{\mathbf{k}+\mathbf{q}=\mathbf{k}_{\mathbf{j}}} \frac{\left|\psi_{j}(z)\right|^{2}\left|\psi_{j}\left(z^{\prime}\right)\right|^{2}}{\left|k_{j}\right|} 2 v^{2} F(\mathbf{k}) F(\mathbf{q})\left|C^{+}\right|^{2}|\Omega|^{2} \mathrm{~d}^{2} \mathbf{k} \mathrm{d}^{2} \mathbf{q} .
$$

This is the same as eq. (51) in Waxler \& Gilbert (2006), except for the fact that we did not replace $\mathbf{q}$ with $-\mathbf{k}$, we thus need to compute the air pressure over the source for all $\mathbf{K}=\mathbf{k}_{\mathbf{j}}$. Although $|\mathbf{K}| \ll|\mathbf{k}|$, the approximation $|\mathbf{K}|=\mathbf{0}$, which is only strictly valid for an azimuth angle $\theta_{a}=0$, leads to very large differences in the source amplitude, up to $30 \mathrm{~dB}$ as shown in Fig. 3. 